REPRESENTATION THEORY

An Electronic Journal of the American Mathematical Society

Volume 15, Pages 646-669 (September 26, 2011)

S 1088-4165(2011)00397-6

\title{
DERIVATIVES AND ASYMPTOTICS OF WHITTAKER FUNCTIONS
}

\author{
NADIR MATRINGE
}

\begin{abstract}
Let $F$ be a $p$-adic field and $G_{n}$ be one of the groups $G L(n, F)$, $G S O(2 n-1, F), G S p(2(n-1), F)$, or $G S O(2(n-1), F)$. Using the mirabolic subgroup or analogues of it, and related "derivative" functors, we give an asymptotic expansion of functions in the Whittaker model of generic representations of $G_{n}$, with respect to a minimal set of characters of subgroups of the maximal torus. Denoting by $Z_{n}$ the center of $G_{n}$ and by $N_{n}$ the unipotent radical of its standard Borel subgroup, we characterize generic representations occurring in $L^{2}\left(Z_{n} N_{n} \backslash G_{n}\right)$ in terms of these characters.

This is related to a conjecture of Lapid and Mao for general split groups, asserting that the generic representations occurring in $L^{2}\left(Z_{n} N_{n} \backslash G_{n}\right)$ are the generic discrete series; we prove it for the group $G_{n}$.
\end{abstract}

\section{INTRODUCTION}

Let $G_{n}$ be the points of one of the groups $G L(n), G S O(2 n-1), G S p(2 n)$, or $G S O(2(n-1))$ over a $p$-adic field $F$. The main result (Theorem 2.1) of this work describes the asymptotic behaviour of the restriction of Whittaker functions to the standard maximal torus in terms of a family of characters which is minimal in some sense. From results of [L-M], this restriction can be described for split reductive groups in terms of cuspidal exponents.

Here, after having defined analogues of the mirabolic subgroup for the groups $G_{n}$ and the corresponding derivative functors, following [C-P (where the case of completely reducible derivatives is treated for $G L(n)$ ), we choose to describe the restriction of Whittaker functions to the torus in terms of central exponents of the derivatives.

This description, inspired by $\mathrm{B}$, is better adapted to understanding when the Whittaker model of a unitary generic representation is a subspace of $L^{2}\left(Z_{n} N_{n} \backslash G_{n}\right)$ (these representations are conjectured to be generic discrete series by Lapid and Mao).

In the first section, we review the groups in question and define their mirabolic subgroups. We also give a decomposition of the unipotent radical of the standard Borel subgroup and a description of how nondegenerate characters of this radical behave with respect to this decomposition.

In Section 2, we give properties of the derivative functors and use them to prove our asymptotic expansion of Whittaker functions, which is Theorem 2.1

Received by the editors April 7, 2010 and in revised form, September 12, 2010 and October 6, 2010 .

2010 Mathematics Subject Classification. Primary 22E50, 22E35.

This work was supported by the EPSRC grant EP/G001480/1. 
In Section 3, we characterize generic representations with Whittaker model included in $L^{2}\left(Z_{n} N_{n} \backslash G_{n}\right)$ in terms of central exponents of the derivatives, in Corollary [3.1. We then prove in Theorem 3.2 the conjecture 3.5 of [L-M].

After the writing of this paper, Patrick Delorme informed the author that he obtained the proof of the conjecture of Lapid and Mao in the general case (Theorem 8 of [D2]).

The author would like to thank Shaun Stevens for inviting him as a postdoctoral fellow at the University of East Anglia, where this paper was written, and also for useful discussions and for English corrections concerning this work. The author also thanks the referee for suggestions concerning the organisation of the paper.

\section{Mirabolic SUbGroup AND NONDEGENERATE CHARACTERS}

Let $F$ be a nonarchimedean local field. We denote by $\mathfrak{O}_{F}$ its ring of integer and by $\mathfrak{P}_{F}=\varpi_{F} \mathfrak{O}_{F}$ the maximal ideal of this ring, where $\varpi_{F}$ is a uniformiser of $F$.

We give a list of groups and describe some of their properties which will be used in the sequel:

- Case A:

The group $G_{0}$ is trivial and, for $n \geq 1$, the group $G_{n}$ is $G L(n, F)$.

We consider the maximal torus of $G_{n}$ consisting of diagonal matrices; it is isomorphic to $\left(F^{*}\right)^{n}$.

For $n \geq 2$, the simple roots of this group can be chosen to be the characters

$$
\alpha_{i}\left(\operatorname{diag}\left(x_{1}, \ldots, x_{n}\right)\right)=x_{i} x_{i+1}^{-1}
$$

for $i$ between 1 and $n-1$.

The root subgroup $U_{\alpha_{i}}$ is given by matrices of the form $I_{n}+x E_{i, i+1}$ for $x$ in $F$.

Standard Levi subgroups of $G_{n}$ are given by matrices of the form $\operatorname{diag}\left(a_{1}, \ldots, a_{r}\right)$ where $a_{i}$ belongs to $G L\left(n_{i}, F\right)$, with $n_{1}+\cdots+n_{r}=n$. We denote the preceding group by $M_{\left(n_{1}, \ldots, n_{r}\right)}$, and the corresponding standard parabolic subgroup is denoted by $P_{\left(n_{1}, \ldots, n_{r}\right)}$, with unipotent radical $U_{\left(n_{1}, \ldots, n_{r}\right)}$.

For $n \geq 2$ we denote by $U_{n}$ the group $U_{(n-1,1)}$ of matrices of the form

$$
\left[\begin{array}{ll}
I_{n-1} & V \\
& 1
\end{array}\right] \text {. }
$$

It is isomorphic to $F^{n-1}$.

For $n>k \geq 1$, the group $G_{k}$ embeds naturally in $G_{n}$ and is given by matrices of the form $\operatorname{diag}\left(g, I_{n-k}\right)$; we denote by $Z_{k}$ its center.

We denote by $P_{n}$ the mirabolic subgroup $G_{n-1} \ltimes U_{n}$ of $G_{n}$.

\section{- Case B:}

The group $G_{0}$ is trivial, and the group $G_{1}$ is $F^{*}$.

For $n \geq 2$, the group $G_{n}=G O(2 n-1, F)$ is the group of matrices $g$ in $G L(2 n-1, F)$ such that ${ }^{t} g J g$ belongs to $F^{*} J$, where $J$ is the antidiagonal matrix of $G L(2 n-1, F)$ with ones on the second diagonal. We call the multiplier of an element $g$ in $G_{n}$ the scalar $\mu(g)$ such that ${ }^{t} g J g$ is equal to $\mu(g) J$ (one checks that for this group, the multiplier actually belongs to $\left.F^{*^{2}}\right)$. It is the direct product of $S O(2 n-1, F)$ with $F^{*}$, more precisely, of $S O(2 n-1, F)$ and the group $I\left(F^{*}\right)$ of matrices $I(t)=t I_{2 n-1}$ for $t$ in $F^{*}$. 
The maximal torus of $G_{n}$ is equal to the product of the torus of matrices of the form $\operatorname{diag}\left(x_{n-1}^{-1}, \ldots, x_{1}^{-1}, 1, x_{1}, \ldots, x_{n-1}\right)$ with $I\left(F^{*}\right)$ and is isomorphic to $\left(F^{*}\right)^{n}$.

For $n \geq 3$, the simple roots of this group can be chosen to be the characters

$$
\alpha_{i+1}\left(\operatorname{diag}\left(t x_{n-1}^{-1}, \ldots, t x_{1}^{-1}, t, t x_{1}, \ldots, t x_{n-1}\right)\right)=x_{i} x_{i+1}^{-1},
$$

for $i$ between 1 and $n-2$, and $\alpha_{1}\left(\operatorname{diag}\left(t x_{n-1}^{-1}, \ldots, t x_{1}^{-1}, t, t x_{1}, \ldots, t x_{n-1}\right)\right)=$ $x_{1}^{-1}$.

The root subgroups $U_{\alpha_{i}}$ are given by matrices of the form

$$
\operatorname{diag}\left(1, \ldots, 1, u, 1, \ldots, 1, u^{-1}, 1, \ldots, 1\right)
$$

for matrices $u$ in the unipotent radical of the Borel of $G L(2, F)$.

For $n>k \geq 1$, the group $G_{k}$ embeds naturally in $G_{n}$ and is given by matrices of the form $\operatorname{diag}\left(\mu(g) I_{n-k}, g, I_{n-k}\right)$, where $\mu(g)$ is the multiplier of the element $g$ of $G_{k}$.

Its center $Z_{k}$ is given by matrices of the form

$$
z_{k}(t)=\operatorname{diag}\left(t^{2} I_{n-k}, t I_{2 k-1}, I_{n-k}\right)
$$

for $t$ in $K^{*}$.

For $n \geq 1$, the standard Levi subgroups of $G_{n}$ are given by matrices of the form

$$
\operatorname{diag}\left(\mu(g)^{\tau} a_{r-1}^{-1}, \ldots, \mu(g)^{\tau} a_{1}^{-1}, g, a_{1}, \ldots, a_{r}\right),
$$

where $a_{i}$ belongs to $G L\left(n_{i}, F\right),{ }^{\tau} a$ is the transpose of $a$ with respect to the second diagonal, and $g$ belongs to $G_{m}$, with $2 m-1+2 n_{1}+\cdots+2 n_{r}=2 n-1$. We denote the preceding group by $M_{\left(m ; n_{1}, \ldots, n_{r}\right)}$, and the corresponding standard parabolic subgroup consisting of block upper triangular matrices is denoted by $P_{\left(m ; n_{1}, \ldots, n_{r}\right)}$, with unipotent radical $U_{\left(m ; n_{1}, \ldots, n_{r}\right)}$.

For $n \geq 2$ we denote by $U_{n}$ the group $U_{(n-1 ; 1)}$, of matrices of the form

$$
\left[\begin{array}{ccc}
1 & -{ }^{\tau} V & -\tau V V / 2 \\
& I_{2 n-3} & V \\
& & 1
\end{array}\right]
$$

It is isomorphic to $F^{2 n-3}$.

For $n \geq 2$, we denote by $P_{n}$ the "mirabolic" subgroup $G_{n-1} \ltimes U_{n}$ of $G_{n}$. - Case C:

The group $G_{0}$ is trivial, and the group $G_{1}$ is $F^{*}$.

For $n \geq 2$, the group $G_{n}=G S p(2(n-1), F)$, where $G S p(2(n-1), F)$ is the group of matrices $g$ in $G L(2(n-1), F)$ such that ${ }^{t} g J g$ belongs to $F^{*} J$, where $J=\left[\begin{array}{cc}0 & W \\ -W & 0\end{array}\right]$ and $W$ is the antidiagonal matrix of $G L(n-1, F)$ with ones on the second diagonal. It is the semi-direct product of $S p(2(n-1), F)$ with $F^{*}$, more precisely, of $S p(2 n, F)$ and the group $I\left(F^{*}\right)$ of matrices $I(t)=\operatorname{diag}\left(t I_{n-1}, I_{n-1}\right)$ for $t$ in $F^{*}$.

The maximal torus of $G_{n}$ is equal to the product of the torus of matrices of the form $\operatorname{diag}\left(x_{n-1}^{-1}, \ldots, x_{1}^{-1}, x_{1}, \ldots, x_{n-1}\right)$ with $I\left(F^{*}\right)$ and is isomorphic to $\left(F^{*}\right)^{n}$. 
The simple roots of this group are the characters

$$
\alpha_{i+1}\left(\operatorname{diag}\left(t x_{n-1}^{-1}, \ldots, t x_{1}^{-1}, x_{1}, \ldots, x_{n-1}\right)\right)=x_{i} x_{i+1}^{-1},
$$

for $i$ between 1 and $n-2$, and

$$
\alpha_{1}\left(\operatorname{diag}\left(t x_{n-1}^{-1}, \ldots, t x_{1}^{-1}, x_{1}, \ldots, x_{n-1}\right)\right)=t x_{1}^{-2} .
$$

For $i$ more than 1 , the root subgroup $U_{\alpha_{i}}$ is given by matrices of the form

$$
\operatorname{diag}\left(1, \ldots, 1, u, 1, \ldots, 1, u^{-1}, 1, \ldots, 1\right),
$$

for matrices $u$ in the unipotent radical of the Borel of $G L(2, F)$, whereas $U_{\alpha_{i}}$ is given by matrices $\operatorname{diag}(1, \ldots, 1, u, 1, \ldots, 1)$, for matrices $u$ in the unipotent radical of the Borel of $G L(2, F)$.

For $n>k \geq 2$, the group $G_{k}$ embeds naturally in $G_{n}$ and is given by matrices of the form $\operatorname{diag}\left(\mu(g) I_{n-k}, g, I_{n-k}\right)$, where $\mu(g)$ is the multiplier of the element $g$ of $G_{k}$. Its center $Z_{k}$ is given by matrices of the form $z_{k}(t)=\operatorname{diag}\left(t^{2} I_{n-k}, t I_{2(k-1)}, I_{n-k}\right)$ for $t$ in $F^{*}$. The group $G_{1}$, which is equal to its center $Z_{1}$, embeds as $I\left(F^{*}\right)$.

For $n \geq 1$, the standard Levi subgroups of $G_{n}$ are:

- either matrices

$$
\operatorname{diag}\left(\mu(g)^{\tau} a_{r-1}^{-1}, \ldots, \mu(g)^{\tau} a_{1}^{-1}, g, a_{1}, \ldots, a_{r}\right),
$$

where $a_{i}$ belongs to $G L\left(n_{i}, F\right)$ and $g$ belongs to $G_{m}$ with $m \geq 2$, with $2(m-1)+2 n_{1}+\cdots+2 n_{r}=2(n-1)$. We denote the preceding group by $M_{\left(m ; n_{1}, \ldots, n_{r}\right)}$, and the corresponding standard parabolic subgroup consisting of block upper triangular matrices is denoted by $P_{\left(m ; n_{1}, \ldots, n_{r}\right)}$, with unipotent radical $U_{\left(m ; n_{1}, \ldots, n_{r}\right)}$.

- or the matrices

$$
z_{1} \cdot \operatorname{diag}\left(a_{r-1}^{-1}, \ldots, a_{1}^{-1}, a_{1}, \ldots, a_{r}\right),
$$

with $a_{i}$ in $G L\left(n_{i}, F\right), 2 n_{1}+\cdots+2 n_{r}=2(n-1)$, and $z_{1}$ in $G_{1}$.

For $n \geq 2$, we denote by $U_{n}$ the group $U_{(n-1 ; 1)}$ of matrices of the form

$$
\left[\begin{array}{cccc}
1 & -{ }^{\top} V_{2} & { }^{\tau} V_{1} & x \\
& I_{n-2} & & V_{1} \\
& & I_{n-2} & V_{2} \\
& & & 1
\end{array}\right] .
$$

For $n \geq 3$, it is an extension of $F^{2(n-2)}$ by $F$, which is the Heisenberg group corresponding to the alternating bilinear form on $F^{2(n-2)}$, given by $\left(W_{1}, W_{2}\right) \times\left(V_{1}, V_{2}\right) \mapsto-{ }^{\tau} W_{2} . V_{1}+{ }^{\tau} W_{1} . V_{2}$.

It is a two-step nilpotent subgroup, with center equal to its derived subgroup, given by matrices with $V_{1}=V_{2}=0$, and the maximal abelian quotient $U_{n}^{a b}$ of $U_{n}$ is $F^{2(n-2)}$.

The group $U_{2}$ is the unipotent radical of the standard Borel of $G_{2}=$ $G S p(2, F)=G L(2, F)$ and is isomorphic to $(F,+)$.

For $n \geq 2$, we denote by $P_{n}$ the "mirabolic" subgroup $G_{n-1} \ltimes U_{n}$ of $G_{n}$.

- Case D:

We denote by $G_{0}$ the trivial group. The group $G_{1}$ is $F^{*}$.

For $n \geq 2$, the group $G_{n}=G S O(2(n-1), F)$ is the group of matrices $g$ in $G L(2(n-1), F)$ satisfying the fact that ${ }^{t} g J g$ belongs to $F^{*} J$, where 
$J$ is the antidiagonal matrix of $G L(2(n-1), F)$ with ones on the second diagonal. It is the semi-direct product of $S O(2(n-1), F)$ with the group $I\left(F^{*}\right)$, of the matrices $I(t)=\operatorname{diag}\left(t I_{n-1}, I_{n-1}\right)$ for $t$ in $F^{*}$.

The maximal torus of $G_{n}$ is equal to the product of the torus of matrices of the form $\operatorname{diag}\left(x_{n-1}^{-1}, \ldots, x_{1}^{-1}, x_{1}, \ldots, x_{n-1}\right)$ with $I\left(F^{*}\right)$, and it is isomorphic to $\left(F^{*}\right)^{n}$.

If $n$ is 2 , then $G_{2}$ is the diagonal torus of $G L(2, F)$.

For $n \geq 3$, the simple roots of this group can be chosen to be the characters

$$
\alpha_{i+1}\left(\operatorname{diag}\left(t x_{n-1}^{-1}, \ldots, t x_{1}^{-1}, x_{1}, \ldots, x_{n-1}\right)\right)=x_{i} x_{i+1}^{-1},
$$

for $i$ between 1 and $n-2$, and $\alpha_{1}\left(\operatorname{diag}\left(t x_{n}^{-1}, \ldots, t x_{1}^{-1}, x_{1}, \ldots, x_{n}\right)\right)=$ $t x_{1}^{-1} x_{2}^{-1}$.

For $i \geq 1$, the root subgroup $U_{\alpha_{i+1}}$ is given by matrices of the form

$$
\operatorname{diag}\left(1, \ldots, 1, u, 1, \ldots, 1, u^{-1}, 1, \ldots, 1\right)
$$

for matrices $u$ in the unipotent radical of the Borel of $G L(2, F)$, whereas $U_{\alpha_{1}}$ is given by matrices $\operatorname{diag}(1, \ldots, 1, u, 1, \ldots, 1)$ for matrices $u$ of the form

$$
\left[\begin{array}{cccc}
1 & & y & \\
& 1 & & -y \\
& & 1 & \\
& & & 1
\end{array}\right] \text {, with } y \text { in } F
$$

For $n>k \geq 2$, the group $G_{k}$ embeds naturally in $G_{n}$ and is given by matrices of the form $\operatorname{diag}\left(\mu(g) I_{n-k}, g, I_{n-k}\right)$, where $\mu(g)$ is the multiplier of the element $g$ of $G_{k}$. For $k \geq 3$, its center $Z_{k}$ is given by matrices of the form $z_{k}(t)=\operatorname{diag}\left(t^{2} I_{n-k}, t I_{2(k-1)}, I_{n-k}\right)$ for $t$ in $F^{*}$.

We denote by $Z_{2}$ the subgroup of the torus $G_{2}$, given by matrices of the form $z_{2}(t)=\operatorname{diag}\left(t I_{n-2}, 1, t, I_{n-2}\right)$ for $t$ in $F^{*}$.

The group $G_{1}$ which is equal to its center $Z_{1}$ embeds as $I\left(F^{*}\right)$.

The standard Levi subgroups of $G_{n}$ are the following:

- The groups given by matrices of the form

$$
\operatorname{diag}\left(\mu(g)^{\tau} a_{r}^{-1}, \ldots, \mu(g)^{\tau} a_{1}^{-1}, g, a_{1}, \ldots, a_{r}\right),
$$

where $a_{i}$ belongs to $G L\left(n_{i}, F\right), g$ belongs to $G_{m}$ with $m \geq 3$, with $2(m-1)+2 n_{1}+\cdots+2 n_{r}=2(n-1)$. We denote the preceding group by $M_{\left(m ; n_{1}, \ldots, n_{r}\right)}$, and the corresponding standard parabolic subgroup is denoted by $P_{\left(m ; n_{1}, \ldots, n_{r}\right)}$, with unipotent radical $U_{\left(m ; n_{1}, \ldots, n_{r}\right)}$.

- The groups given by matrices of the form

$$
g_{2} . \operatorname{diag}\left({ }^{\tau} a_{r}^{-1}, \ldots,{ }^{\tau} a_{1}^{-1}, 1,1, a_{1}, \ldots, a_{r}\right),
$$

with $a_{i}$ in $G L\left(n_{i}, F\right), 2 n_{1}+\cdots+2 n_{r}=2(n-2)$, and $g_{2}$ in $G_{2}$.

- The groups given by matrices of the form

$$
z_{1} \cdot \operatorname{diag}\left({ }^{\tau} a_{r}^{-1}, \ldots,{ }^{\tau} a_{1}^{-1}, a_{1}, \ldots, a_{r}\right),
$$

with $a_{i}$ in $G L\left(n_{i}, F\right), 2 n_{1}+\cdots+2 n_{r}=2(n-1)$, and $z_{1}$ in $G_{1}$.

- The groups given by matrices of the form

$$
z_{2} \cdot \operatorname{diag}\left({ }^{\tau} a_{r}^{-1}, \ldots,{ }^{\tau} a_{1}^{-1}, g, a_{1}, \ldots, a_{r}\right),
$$


where $a_{i}$ belongs to $G L\left(n_{i}, F\right)$, and $g$ in $G L(2(m-1), F)$ is of the form

$$
\left[\begin{array}{cccc}
A & & V & \\
& t^{\prime} & & L^{\prime} \\
L & & t & \\
& V^{\prime} & & A^{\prime}
\end{array}\right],
$$

with

$$
\left[\begin{array}{ll}
A & V \\
L & t
\end{array}\right] \in G L(m-1, F)
$$

and $A$ a $(m-2 \times m-2)$-matrix,

$$
\left[\begin{array}{cc}
{ }^{\tau} A^{\prime} & { }^{\tau} L^{\prime} \\
{ }^{V^{\prime}} & t^{\prime}
\end{array}\right]=\left[\begin{array}{cc}
A & V \\
L & t
\end{array}\right]^{-1}
$$

with $2 m+2 n_{1}+\cdots+2 n_{r}=2 n$ and $z_{2}$ in $Z_{2}$.

For $n \geq 3$ we denote by $U_{n}$ the subgroup $U_{(n-1 ; 1)}$ of $G_{n}$, of matrices of the form

$$
\left[\begin{array}{ccc}
1 & -\tau & -\tau V V / 2 \\
& I_{2(n-2)} & V \\
& & 1
\end{array}\right] .
$$

It is isomorphic to $F^{2 n-2}$.

For $n \geq 3$ denote by $P_{n}$ the "mirabolic" subgroup $G_{n-1} \ltimes U_{n}$ of $G_{n}$.

We denote by $U_{2}$ the group $U_{\alpha_{1}}$, and by $P_{2}$ the group $G_{1} \ltimes U_{2}$ of $G_{2}$.

Lemma 1.1. We denote by $Z_{i}$ the center of $G_{i}$, except in Case $D$ when $n=2$, where we denote by $Z_{2}$ the subgroup $\operatorname{diag}(1, t)$ with $t$ in $F^{*}$ of $G_{2}$. In all cases, one checks that the maximal torus $A_{n}$ of $G_{n}$ is the direct product $Z_{1} \cdot Z_{2} \ldots Z_{n-1} \cdot Z_{n}$, and each $Z_{i}$ is isomorphic to $F^{*}$. Moreover, the $i$-th root has the property that $\alpha_{i}\left(z_{1} \ldots z_{n}\right)=z_{i}$; in other words, these coordinates parametrize the torus $A_{n}$ such that simple roots become canonical projections.

The unipotent radical $N_{n+1}$ of the standard Borel subgroup of $G_{n+1}$ is equal to $U_{2} \ldots U_{n+1}$. Let $\theta$ be a nondegenerate character of $N_{n+1}$ (i.e. that restricts nontrivially to any of the simple root subgroups). We denote by $\theta_{i+1}$ the character $\theta_{\mid U_{i+1}}$, except in the Case $\mathrm{D}$, for $i=2$. In this case $U_{3}=U_{\alpha_{1}} \times U_{\alpha_{2}}$, and we denote by $\theta_{3}$ the character $\theta_{3}\left(u_{\alpha_{1}} u_{\alpha_{2}}\right)=\theta\left(u_{\alpha_{2}}\right)$.

Because $\theta$ is trivial on $U_{d e r}$, and according to the description of $U_{d e r}$ in Theorem 4.1 of $[\mathrm{B}-\mathrm{H}]$, the character $\theta_{i+1}$ must be trivial on every root subgroup $U_{\alpha}$ contained in $U_{i+1}$ such that $\alpha$ is not simple. Moreover, for Case $\mathrm{D}, n=2$, the character $\theta_{3}$ is trivial on $U_{\alpha_{1}}$.

Conversely, if a nontrivial character $\theta_{i+1}$ of $U_{i+1}$ is trivial on every $U_{\alpha} \subset U_{i+1}$ which is not simple, and if, in Case $\mathrm{D}, n=2$, we impose in addition that $\theta_{3}$ is trivial on $U_{\alpha_{1}}$, then one checks that the normalizer of $\theta_{i+1}$ in the mirabolic subgroup $P_{i+1}$ is $P_{i} U_{i+1}$. As the group $U_{2} \ldots U_{i}$ is a subgroup of $P_{i}$, a family of nontrivial characters $\theta_{i+1}$ of $U_{i+1}$, trivial on every $U_{\alpha} \subset U_{i+1}$ except $U_{\alpha_{i}}$, defines a nondegenerate character of $N_{n+1}=U_{2} \ldots U_{n+1}$ by $\theta\left(u_{2} \ldots u_{n+1}\right)=\prod_{i=1}^{n} \theta_{i+1}\left(u_{i+1}\right)$.

Now we fix such a nondegenerate character $\theta$ and write $\theta^{k}$ for the character $\theta_{2} \ldots \theta_{k}$ of $N_{k}$. 


\section{Derivatives AND WhitTAKeR FUnCtions}

If $G$ is an $l$-group, we denote by $A l g(G)$ the category of smooth complex $G$ modules. If $(\pi, V)$ belongs to $A l g(G), H$ is a closed subgroup of $G$, and $\chi$ is a character of $H$, we denote by $V(H, \chi)$ the subspace of $V$ generated by vectors of the form $\pi(h) v-\chi(h) v$ for $h$ in $H$ and $v$ in $V$. This space is actually stable under the action of the subgroup $N_{G}(\chi)$ of the normalizer $N_{G}(H)$ of $H$ in $G$, which fixes $\chi$.

We denote by $\delta_{H}$ the positive character of $N_{G}(H)$ such that if $\mu$ is a right Haar measure on $G$ and $\lambda$ is the left translation of smooth functions with compact support on $G$, then $\mu \circ \lambda\left(n^{-1}\right)=\delta_{H}(n) \mu$ for $n$ in $N$.

This gives the spaces $V(H, \chi)$ and $V_{H, \chi}=V / V(H, \chi)$ (that we simply denote by $V_{H}$ when $\chi$ is trivial) a structure of smooth $N_{G}(\chi)$-modules.

Notation being as in the first section, and for $k$ an integer between 2 and $n$, we define the following functors:

- First we recall the definition of the Jacquet functors:

Let $P$ be a parabolic subgroup of $G_{n}$, with Levi subgroup $M$, and unipotent radical $U$.

We denote by $J_{P}$ the functor from $\operatorname{Alg}\left(G_{n}\right)$ to $\operatorname{Alg}(M)$ such that if $(\pi, V)$ is a smooth $G_{n}$-module, we have $J_{P}(V)=V_{U}$, and $M$ acts on $J_{P}(V)$ by $J_{P} \pi(m)(v+V(U, 1))=\delta_{U}(m)^{-1 / 2} \pi(m) v+V(U, 1)$.

- With the same notation, we denote by $i_{P}^{G}$ the functor from $A l g(M)$ to $A l g\left(G_{n}\right)$ such that if $\rho$ is a smooth $M$-module, and $\bar{\rho}$ is the corresponding $P$-module obtained by inflation of $\rho$ to $P$, then $i_{P}^{G}(\rho)$ is the $G_{n}$-module $\operatorname{ind}_{P}^{G_{n}}(\bar{\rho})$, where ind is the usual normalized compact induction.

- The functor $\Phi_{\theta_{k}}^{-}$(denoted $r_{U_{k}, \theta_{k}}$ in section 1 of [B-Z.2]) from $\operatorname{Alg}\left(P_{k}\right)$ to $\operatorname{Alg}\left(P_{k-1}\right)$ such that if $(\pi, V)$ is a smooth $P_{k}$-module, $\Phi_{\theta_{k}}^{-} V=V_{U_{k}, \theta_{k}}$, and $P_{k-1}$ acts on $\Phi_{\theta_{k}}^{-}(V)$ by $\Phi_{\theta_{k}}^{-} \pi(p)\left(v+V\left(U_{k}, \theta_{k}\right)\right)=\delta_{U_{k}}(p)^{-1 / 2} \pi(p)(v+$ $\left.V\left(U_{k}, \theta_{k}\right)\right)$.

- The functor $\Phi_{\theta_{k}}^{+}$(denoted $i_{U_{k}, \theta_{k}}$ in section 1 of [B-Z.2]) from $A l g\left(P_{k-1}\right)$ to $\operatorname{Alg}\left(P_{k}\right)$ such that for $\pi$ in $A l g\left(P_{k-1}\right)$, one has $\Phi_{\theta_{k}}^{+} \pi=i n d_{P_{k-1} U_{k}}^{P_{k}}\left(\delta_{U_{k}}^{1 / 2} \pi \otimes\right.$ $\left.\theta_{k}\right)$, where ind is the usual compact induction.

- The functor $\hat{\Phi}_{\theta_{k}}^{+}\left(I_{U_{k}, \theta_{k}}\right.$ in section 1 of [B-Z.2] from $\operatorname{Alg}\left(P_{k-1}\right)$ to $\operatorname{Alg}\left(P_{k}\right)$ such that for $\pi$ in $A l g\left(P_{k-1}\right)$, one has $\Phi_{\theta_{k}}^{+} \pi=\operatorname{Ind}_{P_{k-1} U_{k}}^{P_{k}}\left(\delta_{U_{k}}^{1 / 2} \pi \otimes \theta_{k}\right)$, where Ind is the usual induction.

- The functor $\Psi^{-}$is the Jacquet functor $J_{U_{k}}$ (denoted $r_{U_{k}, 1}$ in section 1 of [B-Z.2] from $\operatorname{Alg}\left(P_{k}\right)$ to $A l g\left(G_{k-1}\right)$ such that if $(\pi, V)$ is a smooth $P_{k^{-}}$ module, $\Psi^{-} V=V_{U_{k}, 1}$, and $G_{k-1}$ acts on $\Psi^{-}(V)$ by $\Psi^{-} \pi(g)(v)+V\left(U_{k}, 1\right)=$ $\delta_{U_{k}}(g)^{-1 / 2} \pi(g)\left(v+V\left(U_{k}, 1\right)\right)$.

- The functor $\Psi^{+}$(denoted $i_{U_{k}, 1}$ in section 1 of [B-Z.2]) from $A l g\left(G_{k-1}\right)$ to $A l g\left(P_{k}\right)$, such that for $\pi$ in $A l g\left(G_{k-1}\right)$, one has $\Psi^{+} \pi=i n d_{G_{k-1} U_{k}}^{P_{k}}\left(\delta_{U_{k}}^{1 / 2} \pi \otimes\right.$ 1) $=\delta_{U_{k}}^{1 / 2} \pi \otimes 1$.

As we already fixed the character $\theta$ of $N_{n}$, we will most of the time forget the dependence in $\theta_{k}$ of $\Phi_{\theta_{k}}^{-}$and $\Phi_{\theta_{k}}^{+}$, and we will write these functors $\Phi^{-}$and $\Phi^{+}$. These functors have the following properties which follow (except for c) an d), which are trivial) from Proposition 1.9 of $[\mathrm{B}-\mathrm{Z} .2$ : 
Proposition 2.1. a) The functors $\Phi^{-}, \Phi^{+}, \Psi^{-}$, and $\Psi^{+}$are exact.

b) $\Psi^{-}$is left adjoint to $\Psi^{+}$.

$\left.\mathrm{b}^{\prime}\right) \Phi^{-}$is left adjoint to $\hat{\Phi}^{+}$.

c) $\Phi^{-} \Psi^{+}=0$

d) $\Psi^{-} \Psi^{+}=I d$.

Now we want to know how these functors restrict to smooth $P_{k}$-modules which are submodules of the space $C^{\infty}\left(N_{k} \backslash P_{k}, \theta^{k}\right)=\operatorname{Ind}_{N_{k}}^{P_{k}}\left(\theta^{k}\right)$ of functions on $P_{k}$, fixed by some open subgroup of $P_{k}$ under right translation, and which transform by $\theta^{k}$ under left translation by elements of $N_{k}$.

The next proposition shows the stability of this type of module under $\Phi^{-}$and $\Phi^{+}$.

Proposition 2.2. For any submodule $\tau$ of $C^{\infty}\left(N_{k} \backslash P_{k}, \theta^{k}\right)$, the $P_{k-1}$-module $\Phi^{-} \tau$ is a submodule of $C^{\infty}\left(N_{k-1} \backslash P_{k-1}, \theta^{k-1}\right)$, with model given by restriction of functions $\delta_{U_{k}}^{-1 / 2} W$ in $\tau$ to $P_{k-1}$, and such that we have $\Phi^{-} \tau(p) W=\rho(p) W$ for $p$ in $P_{k-1}$, where $\rho$ is the action by right translation.

Conversely, the $P_{k+1}$-module $\Phi^{+} \tau$ can be identified with a submodule of $C^{\infty}\left(N_{k+1} \backslash P_{k+1}, \theta^{k+1}\right)$, with the natural action of $P_{k+1}$ by right translation.

Proof. The first property will hold if we show that $C^{\infty}\left(N_{k} \backslash P_{k}, \theta^{k}\right)\left(U_{k}, \theta_{k}\right)$ is the kernel of the restriction map to $C^{\infty}\left(N_{k-1} \backslash P_{k-1}, \theta^{k-1}\right)$. This is a straightforward adaptation of the proof of Proposition 2.1 of C-P.

The second property is a consequence of the following equalities and inclusions:

$$
\begin{aligned}
\Phi^{+}\left(C^{\infty}\left(N_{k} \backslash P_{k}, \theta^{k}\right)\right) & =\operatorname{ind}_{P_{k} U_{k+1}}^{P_{k+1}}\left(\delta_{U_{k+1}}^{1 / 2} \cdot \operatorname{Ind}_{N_{k}}^{P_{k}}\left(\theta^{k}\right) \otimes \theta_{k+1}\right) \\
& \subset \operatorname{Ind}_{P_{k} U_{k+1}}^{P_{k+1}}\left(\delta_{U_{k+1}}^{1 / 2} \cdot \operatorname{Ind}_{N_{k}}^{P_{k}}\left(\theta^{k}\right) \otimes \theta_{k+1}\right) .
\end{aligned}
$$

Then

$$
\delta_{U_{k+1}}^{1 / 2} \cdot \operatorname{Ind}_{N_{k}}^{P_{k}}\left(\theta^{k}\right) \simeq \operatorname{Ind}_{N_{k}}^{P_{k}}\left(\theta^{k}\right)
$$

because the character $\delta_{U_{k+1}}^{1 / 2}$ of $P_{k}$ is trivial on $N_{k}$.

Finally,

$$
\operatorname{Ind}_{P_{k} U_{k+1}}^{P_{k+1}}\left(\operatorname{Ind}_{N_{k}}^{P_{k}}\left(\theta^{k}\right) \otimes \theta_{k+1}\right) \simeq \operatorname{Ind}_{N_{k+1}}^{P_{k+1}}\left(\theta^{k+1}\right) .
$$

More can be said about smooth $P_{k}$-submodules of the space $C^{\infty}\left(N_{k} \backslash P_{k}, \theta^{k}\right)=$ $\operatorname{Ind}_{N_{k}}^{P_{k}}\left(\theta^{k}\right)$. If $\tau$ is a $P_{k^{-}}$-submodule of $C^{\infty}\left(N_{k} \backslash P_{k}, \theta^{k}\right)$, then the derived subgroup of $U_{k}$ (which is trivial except in Case D) acts trivially.

To see this, take $W$ in $C^{\infty}\left(N_{k} \backslash P_{k}, \theta^{k}\right)$. We claim that if $u$ belongs to the derived subgroup $U_{k}^{d e r}$ of $U_{k}$, then $\tau(u) W$ and $W$ are equal. Hence let $p$ belong to $P_{k}$; one has $\tau(u) W(p)=W(p u)=W\left(p u p^{-1} p\right)=\theta^{k}\left(p u p^{-1}\right) W(p)$. However, $P_{k}$ normalizes $U_{k}\left(\right.$ so $\theta^{k}\left(\right.$ pup $\left.^{-1}\right)=\theta_{k}\left(\right.$ pup $\left.\left.^{-1}\right)\right)$ so that it normalizes its derived subgroup as well; as $\theta_{k}$ is trivial on this subgroup, this proves our claim.

For such modules being $P_{k}$-modules, there is a nice interpretation of $V\left(U_{k}, 1\right)$ in terms of the analytic behaviour of Whittaker functions. First, we make the following observation.

Remark 2.1. For $k \geq 3$, as a consequence of the Iwasawa decomposition, any element $g$ of $G_{k-1}$ can be written in the form $p z c$ with $p$ in $P_{k-1}, z$ in $Z_{k-1}$, and $c$ in $K=G_{k-1}\left(\mathfrak{O}_{F}\right)$, and the absolute value of $z$ depends only on $g$, so we can write it $|z(g)|_{F}$. 
If a function $W$ is in the space of $C^{\infty}\left(N_{k} \backslash P_{k}, \theta^{k}\right)$, then for $g$ in $G_{k-1}$ we show that $W(g)$ vanishes whenever $|z(g)|_{F}$ is large enough.

Indeed if we take the "natural" group isomorphism $u$ from $\left(F^{m},+\right)$ to $U_{k}^{a b}$, for some positive integer $m$, and recalling that it is in fact $U_{k}^{a b}$ that acts on $V$, then $u(x)$ will fix $W$ for $x$ near zero in $F^{m}$.

But then, for $g$ in $G_{k-1}$ of the form $p z c$, one has $W(g)=W(g u(x))=$ $\theta_{k}\left(g u(x) g^{-1}\right) W(g)$, which is equal to $\theta_{k}\left(z c u(x)(c z)^{-1}\right) W(g)$ because $P_{k-1}$ normalizes $\theta_{k}$. This implies the equality $\left[\theta_{k}\left(z c u(x)(c z)^{-1}\right)-1\right] W(g)=\left[\theta_{k}(u(z c x))-\right.$ $1] W(g)=0$ for any $x$ in a neighbourhood of zero depending only on $W$. The assertion follows easily.

Proposition 2.3. Let $(\tau, V)$ be a $P_{k}$-submodule of $C^{\infty}\left(N_{k} \backslash P_{k}, \theta^{k}\right)$. Then the space $V\left(U_{k}, 1\right)$ is the subspace of $V$, of functions $W$ such that there exists an integer $N_{W}$ with $W(g)=0$, for any $g$ satisfying $|z(g)|_{F} \leq q_{F}^{-N_{W}}$.

Proof. Suppose first that a function $W$ is in $V\left(U_{k}, 1\right)$, so we can write it $\pi(u) W^{\prime}-W^{\prime}$ for some $u$ in $U_{k}^{a b}$ and some $W^{\prime}$ in $V$. Then, writing $g$ as $p z c$ and $u$ as $u(x)$ for $x$ in $F^{m}$, we have $\left[\pi(u) W^{\prime}-W^{\prime}\right](g)=\left[\theta_{k}(u(z c x))-1\right] W^{\prime}(g)$, which will be zero to 0 when $|z|_{F}$ is close to zero.

Conversely, we use the characterization of Jacquet and Langlands, asserting that the elements $W$ of $V\left(U_{k}, 1\right)=V\left(U_{k}^{a b}, 1\right)$ are those such that $\int_{U} \tau(u) W d u$ is zero as soon as the open compact subgroup $U$ of $U_{k}^{a b}$ contains some compact open subgroup $U_{W}$. Therefore, suppose $W$ is in $V$ and that it vanishes on elements $g$ of $G_{n-1}(F)$ satisfying $|z(g)|_{F} \leq q_{F}^{-N_{W}}$.

Let $U$ be any open compact subgroup of $U_{k}^{a b}$ that we identify with a subgroup of $F^{m}$. The integral $\int_{U} \tau(u) W d u$ evaluated at $g=p z c$ is equal to $\int_{x \in U} \theta_{k}(z c x) W(g) d x$. Hence this integral is always zero for $|z|_{F} \leq q_{F}^{-N_{W}}$ because $W(g)$ is as well.

We now recall that as $\theta_{k}$ is a nontrivial character of $U_{k}^{a b}$, there exists a compact open ball $U_{0}$ of $U_{k}^{a b} \simeq F^{n}$ such that the integral $\int_{x \in U} \theta_{k}(x) d x$ is zero whenever the compact open subgroup $U$ of $U_{k}^{a b}$ contains $U_{0}$. However, then for $|z|_{F} \geq q_{F}^{-N_{W}}$, if $t_{W}$ is an element of $F^{*}$ of absolute value $q_{F}^{N_{W}}$, the integral $\int_{x \in U} \theta_{k}(z c x) W(g) d x$ is also zero as soon as $U$ contains $t_{W} U_{0}$. Hence $\int_{U} \tau(u) W d u$ is zero when $U$ is a compact open subgroup of $U_{k}^{a b}$ containing $U_{W}=t_{W} U_{0}$ and when $W$ belongs to $V\left(U_{k}, 1\right)$.

For any smooth $P_{n}$-module $\tau$ and any integer $k \geq 1$, we denote by $\tau_{(k)}$ the representation of $P_{n-k+1}$ equal to $\left(\Phi^{-}\right)^{k-1} \tau$, and by $\tau^{(k)}$ the representation of $G_{n-k}$ equal to $\Psi^{-}\left(\Phi^{-}\right)^{k-1} \tau=\Psi^{-} \tau_{(k)}$.

We say that a smooth irreducible representation $\pi$ of $G_{n}$ is $\theta^{n}$-generic if it is isomorphic to a submodule of the induced representation $\operatorname{Ind}_{N_{n}}^{G_{n}}\left(\theta^{n}\right)$. If it is the case, the submodule of $\operatorname{Ind}_{N_{n}}^{G_{n}}\left(\theta^{n}\right)$ isomorphic to $\pi$ is unique, and it is called the Whittaker model of $\pi$ and is denoted by $W\left(\pi, \theta^{n}\right)$.

Now we let $(\pi, V)$ be a $\theta^{n}$-generic representation of $G_{n}$ (hence a smooth $P_{n^{-}}$ module as well). We denote by $\left(\pi^{\prime}, V^{\prime}\right)$ the representation of $P_{n}$ obtained on the space of restrictions of functions in $W(\pi, \theta)$ to $P_{n}$, it is a quotient of $\pi$ as a $P_{n^{-}}$ module, and restriction to $P_{n}$ is known to be an isomorphism in Case A.

The following proposition follows from repeatedly applying Proposition 2.2. and from Proposition 2.3 . 
Proposition 2.4. Let $\tau$ be a smooth $P_{n}$-submodule of $C^{\infty}\left(N_{n} \backslash P_{n}, \theta^{n}\right)$, and let $k \geq 0$ be an integer. Then the $P_{k+1}$-module $\tau_{(n-k-1)}$ is a submodule of $C^{\infty}\left(N_{k+1} \backslash P_{k+1}, \theta^{k+1}\right)$, with model given by restriction of functions $\left[\delta_{U_{k+2}} \ldots \delta_{U_{n}}\right]^{-1 / 2} W$ in $\tau$ to $P_{k+1}$. In this realisation, one has $\tau_{(n-k-1)}(p) W=$ $\rho(p) W$ for $p$ in $P_{k+1}$, where $\rho$ is the action by right translation.

The next proposition asserts amongst other things that for every $k \geq 1$, the $G_{n-k}$-module $\pi^{(k)}$ has finite length.

Proposition 2.5. If $(\pi, V)$ is a smooth representation of $G_{n}$ of finite length, then for $k$ between 1 and $n-1$, the $G_{k}$-module $\pi^{(n-k)}$ (hence its quotient $\pi^{\prime(n-k)}$ ) has finite length.

Proof. For $k \geq 1$, except in Case D where $k=2$, we denote by $U_{k, n-k}$ the unique standard unipotent radical (denoted by $U_{(k ; n-k)}$ in the previous section) containing $U_{\alpha_{k}}$ as only simple root subgroup.

In Case $\mathrm{D}$, for $k=2$, we denote by $U_{2, n-2}$ the unique standard unipotent radical containing $U_{\alpha_{1}}$ and $U_{\alpha_{2}}$ as only simple root subgroups.

In all cases, the corresponding Levi $M_{k, n-k}$ is the direct product of $G_{k}$ with $G L(n-k, F)$.

Now the $G_{k}$-module $\pi^{(n-k)}$ is a quotient of the Jacquet $G_{k} \times G L(n-k)$-module

$$
\left(\pi_{U_{k, n-k}}, V / V\left(U_{k, n-k}, 1\right)\right),
$$

as the kernel of the surjective map $\pi \rightarrow \pi^{(n-k)}$ contains $V\left(U_{k, n-k}, 1\right)$. More precisely, let $N_{n-k, A}$ be the unipotent radical of the standard Borel subgroup of $G L(n-k, F)$. The group $U_{k+1} \ldots U_{n}$ is the semidirect product $N_{n-k, A} \ltimes U_{k, n-k}$ so that the space $V^{(n-k)}$ of $\pi^{(n-k)}$ is equal to the quotient

$$
V / V\left(N_{n-k, A} \ltimes U_{k, n-k}, \theta_{\mid N_{n-k, A}}^{n} \otimes 1_{U_{k, n-k}}\right),
$$

where $V$ is the space of $\pi$.

We denote by $I_{k}$ the surjection obtained by factorisation from $V_{U_{k, n-k}}$ onto $V^{(n-k)}$. From Lemma 2.32 of [B-Z], the map $I_{k}$ identifies with the projection

$$
V_{U_{k, n-k}} \rightarrow\left(V_{U_{k, n-k}}\right)_{N_{n-k, A}, \theta_{\mid N_{n-k, A}}^{n}}=V_{U_{k, n-k}} / V_{U_{k, n-k}}\left(N_{n-k, A}, \theta_{\mid N_{n-k, A}}^{n}\right) .
$$

The map $I_{k}$ is in fact a $G_{k}$-module morphism because of the equality of modulus characters

$$
\left(\delta_{U_{k, n-k}}\right)_{\mid G_{k}}=\left(\delta_{U_{k+1}} \ldots \delta_{U_{n}}\right)_{\mid G_{k}}
$$

which is itself a consequence of the decomposition

$$
U_{k, n-k}=\prod_{i=k+1}^{n}\left(U_{k, n-k} \cap U_{i}\right) .
$$

The group $N_{A, n-k}$ being a union of compact subgroups, the map $I_{k}$ preserves exact sequences.

As the Jacquet module functor preserves finite length, the $G_{k} \times G L(n-k, F)$ module $\pi_{U_{k, n-k}}$ has a finite composition series $0 \subset\left(\pi_{U_{k, n-k}}\right)_{1} \subset \cdots \subset\left(\pi_{U_{k, n-k}}\right)_{r_{k}}=$ $\pi_{U_{k, n-k}}$. We put $\pi_{i}^{(n-k)}=I_{k}\left[\left(\pi_{U_{k, n-k}}\right)_{i}\right]$.

Hence $\pi_{i}^{(n-k)} / \pi_{i-1}^{(n-k)}$ is equal to $\left[\left(\pi_{U_{k, n-k}}\right)_{i} /\left(\pi_{U_{k, n-k}}\right)_{i-1}\right]_{N_{n-k, A}, \theta_{\mid N_{n-k}, A}}$, but as a $G_{k} \times G L(n-k, F)$-module, the quotient $\left(\pi_{U_{k, n-k}}\right)_{i} /\left(\pi_{U_{k, n-k}}\right)_{i-1}$ isomorphic to $\rho_{1} \otimes \rho_{2}$ for irreducible representations $\rho_{1}$ and $\rho_{2}$ of $G_{k}$ and $G L(n-k, F)$, respectively. 
Because the character $\theta^{n}$ restricts to $N_{n-k, A}$ as a nondegenerate character, the

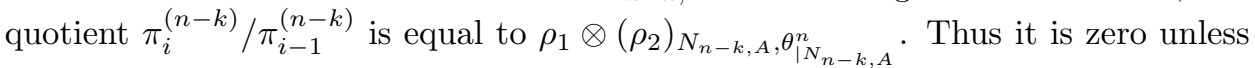
$\rho_{2}$ is generic, in which case it is equal to the irreducible representation $\rho_{1}$.

Therefore we proved that $\pi^{(n-k)}$ has finite length as a $G_{k}$-module, smaller than the length of the Jacquet module $\pi_{U_{k, n-k}}$ as a $G_{k} \times G L(n-k, F)$-module.

There is another property of the maps $I_{k}$ defined in the proof of the preceding proposition that is worth mentioning, that being that their restriction to generalised characteristic subspaces is nonzero. More formally, let $G$ be an $l$-group, and $T$ be a closed abelian subgroup of $G$. If $V$ is a smooth $G$-module, following [C], we define for each character $\chi$ of $T$ the $T$-submodule

$$
V_{\chi, \infty}=\left\{v \in V \mid \exists n \in \mathbb{N}, \forall t \in T,(\tau(t)-\chi(t) I d)^{n}(v)=0\right\} .
$$

If $V$ is $T$-finite (i.e. every vector in $V$ generates a finite dimensional $T$-module), then it is the finite direct sum of its (nonzero by definition) generalised characteristic subspaces, and every such (nonzero) $V_{\chi, \infty}$ contains the nonzero generalised eigenspace

$$
V_{\chi}=\{v \in V \mid \exists n \in \mathbb{N}, \forall t \in T,(\tau(t)-\chi(t) I d)(v)=0\} .
$$

First recall that smooth $\left(F^{*}\right)^{r}$-modules $E$, with a filtration $0=E_{0} \subset E_{1} \subset$ $\cdots \subset E_{r-1} \subset E_{r}=E$ such that $\left(F^{*}\right)^{r}$ acts by a character on each quotient, are $\left(F^{*}\right)^{r}$-finite.

Lemma 2.1. Let $E$ be a smooth $\left(F^{*}\right)^{r}$-module $E$, with a filtration $0=E_{0} \subset E_{1} \subset$ $\cdots \subset E_{r-1} \subset E_{r}=E$ such that $\left(F^{*}\right)^{r}$ acts by a character $c_{i+1}$ on each quotient $E_{i+1} / E_{i}$. Then any vector of $E$ lies in a finite dimensional $\left(F^{*}\right)^{r}$-submodule.

Proof. One proves this by induction on the smallest $i$ such that $E_{i}$ contains $v$. If this $i$ is 1 , the group $\left(F^{*}\right)^{r}$ only multiplies $v$ by a scalar, and we are done.

Suppose that the result is known for $E_{i}$, and take $v$ in $E_{i+1}$ but not in $E_{i}$. Then for every $t$ in $\left(F^{*}\right)^{r}$, the vector $\tau(t) v-c_{i+1}(t) v$ belongs to $E_{i}$. By smoothness, the set $\left\{\tau(u) v \mid u \in\left(U_{F}\right)^{r}\right\}$ is actually equal to $\{\tau(u) v \mid u \in P\}$ for $P$ a finite set of $\left(U_{F}\right)^{r}$. The vector space generated by this set is stabilized by $\left(U_{F}\right)^{r}$ and has a finite basis $v_{1}, \ldots, v_{m}$. Now the vectors

$$
\tau\left(1, \ldots, 1, \varpi_{F}, 1, \ldots, 1\right) v_{l}-c_{i+1}\left(1, \ldots, 1, \varpi_{F}, 1, \ldots, 1\right) v_{l}
$$

belong to $E_{i}$, hence by induction hypothesis to a finite dimensional $\left(F^{*}\right)^{r}$-submodule $V_{l}$ of $E_{i}$. Finally the finite dimensional space $\operatorname{Vect}\left(v_{1}, \ldots, v_{m}\right)+V_{1}+\cdots+V_{m}$ is stable under $\left(U_{F}\right)^{r}$ and the elements $\left(1, \ldots, 1, \varpi_{F}, 1, \ldots, 1\right)$, hence $\left(F^{*}\right)^{r}$, and contains $v$.

This in particular applies to the $Z_{k} Z_{n}$-module $V_{U_{k, n-k}}$ and the $Z_{k}$-module $V$ described in the proof of Proposition 2.5, as both are respectively $G_{k} \times G L(n-k, F)$ and $G_{k}$-modules of finite length.

Now we can prove the following property of the maps $I_{k}$ :

Proposition 2.6. Let $(\pi, V)$ be a $\theta^{n}$-generic representation of $G_{n}$, and for $k \geq 1$, let $U_{k, n-k}$ and $M_{k, n-k} \simeq G_{k} \times G L(n-k, F)$ be the subgroups of $G_{n}$ defined in the proof of Proposition 2.5. Let $\chi$ be a character of the central subgroup $Z_{k} Z_{n}$ of $M_{k, n-k}$, and denote by the same letter its restriction to the central subgroup $Z_{k}$ of $G_{k}$. If the generalised characteristic subspace $\left(V_{U_{k, n-k}}\right)_{\chi, \infty}$ is nonzero, then 
the map $I_{k}$ restricts nontrivially to $\left(V_{U_{k, n-k}}\right)_{\chi}$. In particular, the space $V_{\chi}^{(n-k)}$ is nonzero.

Proof. Suppose that the subspace $\left(V_{U_{k, n-k}}\right)_{\chi, \infty}$ of $V_{U_{k, n-k}}$ is nonzero; hence $\left(V_{U_{k, n-k}}\right)_{\chi}$ is nonzero. The space $\left(V_{U_{k, n-k}}\right)_{\chi}$ is an $M_{k, n-k}$-submodule of $V_{U_{k, n-k}}$, so it has finite length. Hence it contains some irreducible representation $\rho_{1} \otimes \rho_{2}$ of $M_{k, n-k}$. Hence $\operatorname{Hom}_{M_{k, n-k}}\left(\rho_{1} \otimes \rho_{2}, V_{U_{k, n-k}}\right)$ is nonzero, but then from Bernstein's second adjointness theorem (see $[\mathrm{Bu}$, Theorem 3), we deduce that $V$ is a quotient of the representation $\rho_{1} \times \rho_{2}$ parabolically induced from $\rho_{1} \otimes \rho_{2}$. As $V$ admits a nonzero Whittaker form, so does $\rho_{1} \times \rho_{2}$, and from a classical result of Rodier (Theorem 7 of $[\underline{R}]$ ) this implies that $\rho_{1}$ and $\rho_{2}$ are generic with respect to some nondegenrate character. As generiticity doesn't depend on the character for $G L(n-k, F)$, we deuce that $I_{k}\left(\rho_{1} \otimes \rho_{2}\right)=\rho_{1}$. Hence $I_{k}$ restricts nontrivially to $\left(V_{U_{k, n-k}}\right)_{\chi}$, and the image $I_{k}\left[\left(V_{U_{k, n-k}}\right)_{\chi}\right]$ contains $\rho_{1}$, which is a nonzero submodule of $V_{\chi}^{(n-k)}$.

We will also need to know that if $k$ is an integer between 1 and $n-1$ and $\chi$ is a character of $Z_{k}$, then the $Z_{k}$-modules $\left(V^{(n-k)}\right)_{\chi}$ and $\left(V^{\prime(n-k)}\right)_{\chi}$ are nonzero at the same time. We already know from the previous proposition that this is equivalent to the fact that the $Z_{k}$-modules $\left(V^{\prime(n-k)}\right)_{\chi}$ and $\left(V_{U_{k, n-k}}\right)_{\chi}$ are nonzero at the same time, and that $\left(V^{\prime(n-k)}\right)_{\chi}$ nonzero implies that $\left(V_{U_{k, n-k}}\right)_{\chi}$ is nonzero.

Proposition 2.7. If $(\pi, V)$ is a $\theta^{n}$-generic representation of $G_{n}$, and for $k \geq 1$, let $U_{k, n-k}$ and $M_{k, n-k} \simeq G_{k} \times G L(n-k, F)$ be the subgroups of $G_{n}$ defined in the proof of Proposition 2.5. Let $\chi$ be a character of the central subgroup $Z_{k} Z_{n}$ of $M_{k, n-k}$, and denote by the same letter its restriction to the central subgroup $Z_{k}$ of $G_{k}$. Then the space $\left(V_{U_{k, n-k}}\right)_{\chi}$ is nonzero if and only if the space $\left(V^{(n-k)}\right)_{\chi}$ is nonzero.

Proof. We only need to prove that if $\left(V_{U_{k, n-k}}\right)_{\chi}$ is nonzero, then the space $\left(V^{\prime(n-k)}\right)_{\chi}$ is nonzero.

Suppose that the $M_{k, n-k}$-module $\left(V_{U_{k, n-k}}\right)_{\chi}$ is nonzero, it is of finite length, and hence it contains an irreducible $M_{k, n-k}$-submodule $\rho$. Call $P_{k, n-k}$ the parabolic subgroup $M_{k, n-k} U_{k, n-k}$ and $P_{k, n-k}^{-}$its opposite parabolic subgroup (with unipotent radical $\left.\left(U_{k, n-k}\right)^{-}\right)$. We already saw that by Bernstein's second adjointness theorem the induced representation $i_{P_{k, n-k}^{-}}^{G_{n}}(\rho)$ has $\pi$ as a quotient, and therefore $i_{P_{k, n-k}^{-}}^{G_{n}}(\rho)$ is $\theta^{n}$-generic. Then from Theorem 7 of $\left[\mathrm{R}\right.$, the $M_{k, n-k}$-module $\rho$ is $\theta$-generic, where $\theta$ is the restriction of $\theta^{n}$ to the unipotent radical of the Borel of $M_{k, n-k}$. Both have the same Whittaker model $W\left(\pi, \theta^{n}\right)$, i.e. the (unique up to scalar) Whittaker form on the space of $i_{P_{k, n-k}^{-}}^{G_{n}}(\rho)$ factorises through the projection from $i_{P_{k, n-k}^{-}}^{G_{n}}(\rho)$ to $\pi$. Let $L^{-}$be a nonzero $\theta$-Whittaker form on the space of $\rho$. By Theorems 1.4 and 1.6 of [C-S], there is a nonzero Whittaker form $L$ on the space of $i_{P_{k, n-k}^{-}}^{G_{n}}(\rho)$ whose restriction to the subspace $C_{c}^{\infty}\left(P_{k, n-k}^{-} \backslash P_{k, n-k}^{-} U_{k, n-k},\left(\delta_{U_{k}^{-}}\right)^{1 / 2} \rho\right)$ of functions with support in $P_{k, n-k}^{-} U_{k, n-k}$ is given by

$$
f \mapsto \int_{U_{k, n-k}} L^{-}(f(u)) \theta^{-1}(u) d u .
$$


We denote by $\bar{L}$ the Whittaker form on the space of $\pi$ which lifts to $L$. In particular, for any $\bar{f}$ in the space of $\pi$, which is the image of $f$ in $C_{c}^{\infty}\left(P_{k, n-k}^{-} \backslash P_{k, n-k}^{-} U_{k, n-k}\right)$, one has

$$
\bar{L}(\bar{f})=\int_{U_{k, n-k}} L^{-}(f(u)) \theta^{-1}(u) d u .
$$

Let $v$ be a vector in the space of $\rho$ such that $L^{-}(v)$ is nonzero. Let $K^{\prime}$ be a compact subgroup of $G_{n}$, with Iwahori decomposition with respect to $P_{k, n-k}$, and such that $K^{\prime} \cap M_{k, n-k}$ fixes $v$. Then the function $\alpha$ equal to $u^{-} m u \mapsto \rho(m) v$ on $\left(U_{k, n-k}\right)^{-} M_{k, n-k}\left(U_{k, n-k} \cap K^{\prime}\right)$, and zero outside, is well defined and belongs to the space $C_{c}^{\infty}\left(P_{k, n-k}^{-} \backslash P_{k, n-k}^{-} U_{k, n-k}, \rho\right)$. We denote by $W_{\alpha}$ the corresponding Whittaker function $g \mapsto \bar{L}(\pi(g) \bar{\alpha})$. If $a$ belongs to the group $Z_{k}$, one has

$$
\begin{aligned}
W_{\alpha}(a) & =\int_{U_{k, n-k}} L^{-}(\alpha(u a)) \theta^{-1}(u) d u \\
& =\chi(a) \delta_{U_{k, n-k}}^{-1 / 2}(a) \int_{U_{k, n-k}} L^{-}\left(\alpha\left(a^{-1} u a\right)\right) \theta^{-1}(u) d u \\
& =\chi(a) \delta_{U_{k, n-k}}^{1 / 2}(a) \int_{U_{k, n-k}} L^{-}(\alpha(u)) \theta^{-1}\left(a u a^{-1}\right) d u \\
& =\chi(a) \delta_{U_{k, n-k}}^{1 / 2}(a) L^{-}(v) \int_{U_{k, n-k}} \theta^{-1}\left(a u a^{-1}\right) d u .
\end{aligned}
$$

In this last integral, $u$ stays in the compact set $U_{k, n-k} \cap K^{\prime}$. Hence there is a (punctured) neighbourhood of zero in $\operatorname{Lie}\left(Z_{k}\right)=F$ such that $a\left(U_{k, n-k} \cap K^{\prime}\right) a^{-1}$ is a subset of $\operatorname{Ker}(\theta)$ when $a$ belongs to this neighbourhood. Finally, up to multiplication of the function $\alpha$ by a scalar, one has

$$
W_{\alpha}(a)=\chi(a) \delta_{U_{k, n-k}}^{1 / 2}(a) L^{-}(v)
$$

whenever $a$ is this neighbourhood of zero.

A similar computation gives the equality

$$
W_{\alpha}(z g)=\chi(z) \delta_{U_{k, n-k}}(z)^{1 / 2} c_{g}
$$

for $z$ in $Z_{k}$ in a neighbourhood of zero and $g$ in $G_{k}$, where $c_{g}$ is the constant $\int_{U_{k, n-k}} L^{-}(\alpha(u g)) d u$. Hence from Propositions 2.3, 2.4, and the equality $\left(\delta_{U_{k, n-k}}\right)_{\mid G_{k}}$ $=\left(\delta_{U_{k+1}} \ldots \delta_{U_{n}}\right)_{\mid G_{k}}$, we deduce that the vector $\left(\delta_{U_{k+2}} \ldots \delta_{U_{n}}\right)^{-1 / 2} W_{\alpha}$ in the space of $\pi_{(n-k+1)}^{\prime}$ is such that its image in $\pi^{\prime(n-k)}$ is nonzero and belongs to the space $\left(\pi^{\prime(n-k)}\right)_{\chi}$. This proves the proposition.

A straightforward generalisation of the proof of the preceding proposition gives the following corollary.

Corollary 2.1. Let $G$ be the F-points of a quasi-split reductive group defined over $F$. Let $P$ be a parabolic subgroup of $G$ with a Levi subgroup $M$, and let $P^{-}$be its opposite subgroup with $P \cap P^{-}=M$. Let $(\pi, V)$ be a smooth $\theta$-generic representation of $G$, for some nondegenerate character $\theta$ of the unipotent radical $U$ of a Borel subgroup of $G$ contained in $P$. We denote by $j_{P^{-}}$the map defined in Theorem 3.4 of D] from $\left(V^{*}\right)^{U_{\theta}}$ to $\left(J_{P}(V)^{*}\right)^{M \cap U_{\theta}}$. If $L$ is a nonzero vector of the line $\left(V^{*}\right)^{U_{\theta}}$, then the linear form $j_{P^{-}}(L)$ restricts nontrivially to any irreducible $M$-submodule of $J_{P}(V)$ whenever the Jacquet module $J_{P}(V)$ is nonzero. 
We now return to the study of $F^{*}$-modules $E$ with finite factor series such that $F^{*}$ acts by a character on each quotient. From Lemma 2.1, any vector of $E$ will belong to a finite dimensional $F^{*}$-submodule $E^{\prime}$ as in:

Proposition 2.8. If $E^{\prime}$ is a nonzero finite dimensional $F^{*}$-submodule of $E$, then $E^{\prime}$ has a basis $B$ in which the action of $F^{*}$ is given by a block diagonal matrix $M_{B}(\tau(t))$ with each block of the form

$$
\left(\begin{array}{ccccc}
c(t) & c(t) P_{1,2}\left(v_{F}(t)\right) & c(t) P_{1,3}\left(v_{F}(t)\right) & \ldots & c(t) P_{1, q}\left(v_{F}(t)\right) \\
& c(t) & c(t) P_{2,3}\left(v_{F}(t)\right) & \ldots & c(t) P_{2, q}\left(v_{F}(t)\right) \\
& & \ddots & & \vdots \\
& & & c(t) & c(t) P_{q-1, q}\left(v_{F}(t)\right) \\
& & & & c(t)
\end{array}\right)
$$

with $c$ one of the $c_{i}$ 's, $q$ a positive integer depending on the block, and the $P_{i, j}$ 's being polynomials with no constant term of degree at most $j-i$.

Proof. First we decompose $E^{\prime}$ as a direct sum under the action of the compact abelian group $U_{F}$. Because $E^{\prime}$ has a filtration by the spaces $E^{\prime} \cap E_{i}$ and $F^{*}$ acts on each subfactor as one of the $c_{i}$ 's, the group $U_{F}$ acts on each weight space as the restriction of one of the $c_{i}$ 's. Now each weight space is stable under $F^{*}$ by commutativity, and so we can restrict ourselves to the case where $E^{\prime}$ is a weight space of $U_{F}$.

Again $E^{\prime}$ has a filtration such that $F^{*}$ acts on each subfactor as one of the $c_{i}$ 's (with all these characters having the same restriction to $U_{F}$ ), say $c_{i_{1}}, \ldots, c_{i_{k}}$ In particular, we deduce that the endomorphism $\tau\left(\varpi_{F}\right)$ has a triangular matrix in a basis adapted to this filtration, with eigenvalues $c_{i_{1}}\left(\varpi_{F}\right), \ldots, c_{i_{k}}\left(\varpi_{F}\right)$. As $\tau\left(\varpi_{F}\right)$ is trigonalisable, the space $E^{\prime}$ is the direct sum its characteristic subspaces, and again these characteristic subspaces are stable under $F^{*}$.

Finally, one can assume that $E^{\prime}$ is a characteristic subspace for some eigenvalue $c(\pi)$ of $\tau\left(\varpi_{F}\right)$, on which $U_{F}$ acts as the character $c$, where $c$ is one of the $c_{i}$ 's.

Hence there is a basis $B$ of $E^{\prime}$ such that

$$
\operatorname{Mat}_{B}\left(c^{-1}(t) \tau(t)\right)=\left(\begin{array}{ccccc}
1 & A_{1,2}(t) & A_{1,3}(t) & \ldots & A_{1, q}(t) \\
& 1 & A_{2,3}(t) & \ldots & A_{2, q}(t) \\
& & \ddots & & \vdots \\
& & & 1 & A_{q-1, q}(t) \\
& & & & 1
\end{array}\right)
$$

for any $t$ in $F^{*}$, where the $A_{i, j}$ 's are smooth functions on $F^{*}$. Therefore, we only have to prove that the $A_{i, j}$ 's are polynomials of the valuation of $F$ with no constant term.

We do this by induction on $q$. It is obvious when $q=1$. Suppose the statement holds for $q-1$, and suppose that $E^{\prime}$ is of dimension $q$, with basis $B=\left(v_{1}, \ldots, v_{q}\right)$. Considering the two $c^{-1} \tau\left(F^{*}\right)$-modules $\operatorname{Vect}\left(v_{1}, \ldots, v_{q-1}\right)$ and $\operatorname{Vect}\left(v_{1}, \ldots, v_{q}\right) /$ $V e c t\left(v_{1}\right)$ of dimension $q-1$, we deduce that for every couple $(i, j)$ different from $(1, q)$, there is a polynomial with no constant term $P_{i, j}$ of degree at most $j-$ $i$ such that $A_{i, j}=P_{i, j} \circ v_{F}$. Now because $c^{-1} \tau$ is a representation of $F^{*}$ and because the $P_{i, j} \circ v_{F}$ 's vanish on $U_{F}$ for $(i, j) \neq(1, q)$, we deduce that $A_{1, q}$ is a smooth morphism from $\left(U_{F}, \times\right)$ to $(\mathbb{C},+)$, which must be zero because $(\mathbb{C},+)$ has 
no nontrivial compact subgroups. From this we deduce that $A_{1, q}$ is invariant under translation by elements of $U_{F}$ (i.e. $A_{1, q}\left(\varpi_{F}^{k} u\right)=A_{1, q}\left(\varpi_{F}^{k}\right)$ for every $U$ in $\left.U_{F}\right)$.

Denote by $M(k)$ the matrix $M_{B} t_{B}\left(c^{-1} \tau\left(\varpi_{F}^{k}\right)\right)$ for $k$ in $\mathbb{Z}$. One has $M(k)=$ $M(1) M(k-1)$ for $k \geq 1$, which in implies $A_{1, q}\left(\varpi_{F}^{k}\right)=\sum_{j=2}^{q-1} P_{1, j}(1) P_{j, q}(k-1)+$ $A_{1, q}\left(\varpi_{F}^{k-1}\right)+A_{1, q}\left(\varpi_{F}\right)=Q(k)+A_{1, q}\left(\varpi_{F}^{k-1}\right)+A_{1, q}\left(\varpi_{F}\right)$ for $Q$ a polynomial of degree at most $q-2$. This in turn implies that $A_{1, q}\left(\varpi_{F}^{k}\right)=\sum_{l=1}^{k-1} Q(l)+k A_{1, q}\left(\varpi_{F}\right)=$ $R(k)$ for $R$ a polynomial of degree at most $q-1$, according to the theory of Bernoulli polynomials, for any $k \geq 0$. The same reasoning for $k \leq 0$ implies $A_{1, q}\left(\varpi_{F}^{k}\right)=R^{\prime}(k)$ for $R^{\prime}$ a polynomial of degree at most $q-1$, for any $k \leq 0$. We need to show that $R=R^{\prime}$ to conclude.

We know that $M(k)$ is a matrix whose coefficients are polynomials in $k$ for $k>0$ of degree at most $q-1$; we denote it by $P(k)$. The matrix $M(k)$ has the same property for $k<0$; we denote it by $P^{\prime}(k)$. Moreover, for any $k \geq 0$ and $k^{\prime} \leq 0$, with $k+k^{\prime} \geq 0$, one has $P\left(k+k^{\prime}\right)=P(k) P^{\prime}\left(k^{\prime}\right)$. Fix $k>q-1$; then the matrices $P\left(k+k^{\prime}\right)$ and $P(k) P^{\prime}\left(k^{\prime}\right)$ are equal for $k^{\prime}$ in $[1-q, 0]$. As their coefficients are polynomials in $k^{\prime}$ with degree at most $q-1$, the equality $P\left(k+z^{\prime}\right)=P(k) P^{\prime}\left(z^{\prime}\right)$ holds for any complex number $z^{\prime}$. Now fix such a complex number $z^{\prime}$. The equality $P\left(k+z^{\prime}\right)$ and $P(k) P^{\prime}\left(z^{\prime}\right)$ holds for any integer $k>q-1$, and as both matrices have coefficients which are polynomials in $k$, this equality actually holds for any complex number $z$, so that $P\left(z+z^{\prime}\right)$ equals $P(z) P^{\prime}\left(z^{\prime}\right)$ for any complex numbers $z$ and $z^{\prime}$.

As $P(0)=I_{q}$, we deduce that $P$ and $P^{\prime}$ are equal on $\mathbb{C}$, and this implies that $R$ is equal to $R^{\prime}$.

From this we deduce the following theorem, giving an expansion at infinity of Whittaker functions of generic representations of $G_{n}$. For $G L(n)$, the statement holds for any smooth $P_{n}$-submodule of finite length $C^{\infty}\left(N_{n} \backslash P_{n}\right)$ :

Theorem 2.1. Let $\theta$ be a nondegenerate character of the group $N_{n}$, let $\pi$ be a $\theta$-generic representation of $G_{n}$, and let $c_{1, n-k}, \ldots, c_{r_{k}, n-k}$ be the characters of $Z_{k}$ appearing in a composition series of $\tau=\pi^{(n-k)}$. Then, for any function $W$ in the space of $\pi$, the function

$$
W\left(z_{1}, z_{2}, \ldots, z_{n-1}\right)=W\left(z_{1} z_{2} \ldots z_{n-1}\right)
$$

is a linear combination of functions of the form

$$
\prod_{k=1}^{n-1}\left[c_{i_{k}, k} \delta_{U_{k+1}}^{1 / 2} \ldots \delta_{U_{n}}^{1 / 2}\right]\left(z_{k}\right) v_{F}\left(z_{k}\right)^{m_{k}} \phi_{k}\left(z_{k}\right)
$$

for $i_{k}$ between 1 and $r_{k}$, positive integers $m_{k}$, and functions $\phi_{k}$ in $C_{c}^{\infty}(F)$.

Proof. Actually we prove the following stronger statement, which is satisfied by $\pi_{(0)}$ according to Proposition 2.5 .

Let $\pi$ be a submodule of $C^{\infty}\left(N_{n} \backslash P_{n}, \theta\right)$ such that for every $k$ between 1 and $n-1$, the $G_{k^{-}}$module $\tau=\pi^{(n-k)}=\Psi^{-}\left(\Phi^{-}\right)^{n-k-1}(\pi)$ has a composition series such that on each respective quotient, the central subgroup $Z_{k}$ acts by the characters $c_{1, n-k}, \ldots, c_{r_{k}, n-k}$. 
Then, for any function $W$ in the space of $\pi$, the function $W\left(z_{1}, z_{2}, \ldots, z_{n-1}\right)=$ $W\left(z_{1} z_{2}, \ldots, z_{n-1}\right)$ is a linear combination of functions of the form

$$
\prod_{k=1}^{n-1}\left[c_{i_{k}, k} \delta_{U_{k+1}}^{1 / 2} \ldots \delta_{U_{n}}^{1 / 2}\right]\left(z_{k}\right) v_{F}\left(z_{k}\right)^{m_{k}} \phi_{k}\left(z_{k}\right)
$$

for $i_{k}$ between 1 and $r_{k}$, positive integers $m_{k}$, and functions $\phi_{k}$ in $C_{c}^{\infty}(F)$.

We first give the principle of the proof for the group $G_{2}$, which already contains the main ideas. We give the general proof after.

Let $\pi$ be a submodule of $C^{\infty}\left(N_{2} \backslash P_{2}, \theta\right)$ such that $\pi^{(1)}$ has a finite composition series with the group $G_{1}=Z_{1}$ acting by the character $c_{i, 1}$ on the $i$-th quotient. Let $W$ belong to the space of $\pi$ and $v$ be its image in the space $E$ of $\pi^{(1)}$. It belongs to a finite dimensional $Z_{1}$-submodule $E^{\prime}$ of $E$, on which $Z_{1}$ acts by a matrix of the form determined in Proposition 2.8 Take a basis $B=\left(e_{1}, \ldots, e_{q}\right)$ of $E^{\prime}$, and denote by $M(a)$ the matrix $M_{B}(\tau(a))$ (with $a$ in $Z_{1}$ and $\tau(a)=\pi^{(1)}(a)$ ). Hence we have $\tau(a) e_{l}=\sum_{k=1}^{q} M(a)_{k, l} e_{k}$ for each $l$ between 1 and $q$.

Taking preimages $\tilde{E}_{1}, \ldots, \tilde{E}_{q}$ of $e_{1}, \ldots, e_{q}$ in $\pi_{(0)}$, we denote by $\tilde{E}$ the function vector

$$
\left(\begin{array}{c}
\tilde{E}_{1} \\
\vdots \\
\tilde{E}_{q}
\end{array}\right) .
$$

If the image $v$ of $W$ in $\pi^{(1)}$ is equal to $x_{1} e_{1}+\cdots+x_{q} e_{q}$, there is an integer $M$ such that for every $z_{1}$ in $Z_{1}$, the function

$$
W\left(z_{1}\right)-\left(x_{1}, \ldots, x_{q}\right) \tilde{E}\left(z_{1}\right)
$$

vanishes for $\left|z_{1}\right|_{F} \leq q_{F}^{-M}$ according to Proposition 2.3. We denote by $S$ the function $\left(x_{1}, \ldots, x_{q}\right) \tilde{E}$.

Because of Remark 2.1, there is an integer $M^{\prime}$ such that for any $z_{1}$ in $Z_{1}$ of absolute value greater than $q_{F}^{M^{\prime}}$, both $W\left(z_{1}\right)$ and $S\left(z_{1}\right)$ are zero, so that the difference $D\left(z_{1}\right)$ of the two functions is a smooth function which vanishes whenever $z_{1}$ has absolute value outside $\left[q_{F}^{-M}, q_{F}^{M^{\prime}}\right]$.

On the other hand, for any $a$ in $Z_{1}$, one has $\rho(a) p\left(\tilde{E}_{l}\right)=\sum_{k=1}^{q} M(a)_{k, l} p\left(\tilde{E}_{k}\right)$ for $p$ the projection from $\pi_{(0)}$ to $\pi^{(1)}$. As we identified the kernel of this projection in Proposition 2.3, we deduce (see the general proof after) that there is a $z_{0}$ in $Z_{1}$ such that the function $\mathbf{1}_{\left\{\left|z_{1}\right| \leq 1\right\}} S\left(z_{1}\right)$ is equal to

$$
\left(x_{1}, \ldots, x_{q}\right)^{t} M\left(z_{1}\right)\left[\delta_{U_{2}}^{-1 / 2}\left(z_{0}\right)^{t} M\left(z_{0}^{-1}\right)\right] \tilde{E}\left(z_{1}\right) \delta_{U_{2}}^{1 / 2}\left(z_{1}\right) \mathbf{1}_{\left\{\left|z_{1}\right| \leq 1\right\}} .
$$

As for the function $D$, one then proves that there exist two positive integers $M_{1}$ and $M_{1}^{\prime}$ such that the function $\mathbf{1}_{\left\{\left|z_{1}\right|>1\right\}} S\left(z_{1}\right)$ has its support contained in an interval of the form $\left[q_{F}^{-M_{1}}, q_{F}^{M_{1}^{\prime}}\right]$.

Finally the function

$$
W\left(z_{1}\right)=\delta_{U_{2}}^{1 / 2}\left(z_{1}\right)\left[\delta_{U_{2}}^{-1 / 2}\left(z_{1}\right) D\left(z_{1}\right)\right]+\mathbf{1}_{\left\{\left|z_{1}\right| \leq 1\right\}} S\left(z_{1}\right)+\delta_{U_{2}}^{1 / 2}\left(z_{1}\right)\left[\delta_{U_{2}}^{-1 / 2}\left(z_{1}\right) \mathbf{1}_{\left\{\left|z_{1}\right|>1\right\}} S\left(z_{1}\right)\right]
$$

has the desired form because the left and right terms between [ . ] of the sum have compact support in $Z_{1}$ and the middle term is of the requested form according to Proposition 2.8 .

The general proof is by induction on $n$. For $n=1$, the group $G_{1}$ is isomorphic to $F^{*}$, the group $P_{1}$ is trivial, and the statement is obvious. Suppose now that $n$ is 
greater than or equal to 2. Let $W$ belong to the space of $\pi$. We denote by $v$ its image in the space $E$ of $\pi^{(1)}$. The vector $v$ belongs to a finite dimensional $Z_{n-1}$-submodule $E^{\prime}$ of $E$, on which $Z_{n-1}$ acts by a matrix of the form determined in Proposition 2.8. We fix a basis $B=\left(e_{1}, \ldots, e_{q}\right)$ of $E^{\prime}$ and denote by $M(a)$ the matrix $M_{B}(\tau(a))$ (with $a$ in $Z_{n-1}$ and $\tau(a)=\pi^{(1)}(a)$ ). Hence we have $\tau(a) e_{l}=\sum_{k=1}^{q} M(a)_{k, l} e_{k}$ for each $l$ between 1 and $q$.

Taking preimages $\tilde{E}_{1}, \ldots, \tilde{E}_{q}$ of $e_{1}, \ldots, e_{q}$ in $\pi_{(0)}$, we denote by $\tilde{E}$ the function vector

$$
\left(\begin{array}{c}
\tilde{E}_{1} \\
\vdots \\
\tilde{E}_{q}
\end{array}\right)
$$

If the image $v$ of $W$ in $\pi^{(1)}$ is equal to $x_{1} e_{1}+\cdots+x_{q} e_{q}$, there is an integer $M$ such that for every $\left(z_{1}, \ldots, z_{n-2}\right)$ in $Z_{1} \times \cdots \times Z_{n-2}$, the function

$$
W\left(z_{1}, \ldots, z_{n-1}\right)-\left(x_{1}, \ldots, x_{q}\right) \tilde{E}\left(z_{1}, \ldots, z_{n-1}\right)
$$

vanishes for $\left|z_{n-1}\right|_{F} \leq q_{F}^{-M}$. We denote by $S$ the function $\left(x_{1}, \ldots, x_{q}\right) \tilde{E}$.

Because of Remark 2.1, there is an integer $M^{\prime}$ such that for any $\left(z_{1}, \ldots, z_{n-2}\right)$ in $Z_{1} \times \cdots \times Z_{n-2}$ and any $z_{n-1}$ in $Z_{n-1}$ of absolute value greater than $q_{F}^{M^{\prime}}$, both $W\left(z_{1}, \ldots, z_{n-1}\right)$ and $S\left(z_{1}, \ldots, z_{n-1}\right)$ are zero, so that the difference $D\left(z_{1}, \ldots, z_{n-1}\right)$ of the two functions is a smooth function which vanishes whenever $z_{n-1}$ has absolute value outside $\left[q_{F}^{-M}, q_{F}^{M^{\prime}}\right]$. Moreover, there is a compact subgroup $U$ of $Z_{n-1}\left(\mathfrak{O}_{F}\right)$ independent of $\left(z_{1}, \ldots, z_{n-1}\right)$ such that both functions (hence $D$ ) are invariant when $z_{n-1}$ is multiplied by an element of $U$. Denoting by $\left(z_{\alpha}\right)_{\alpha} \in A$ a finite set of representatives for

$$
\left\{\left.z\left|q_{F}^{-M} \leq\right| z_{n-1}\right|_{F} \leq q_{F}^{M^{\prime}}\right\} / U
$$

this implies that $D\left(z_{1}, \ldots, z_{n-1}\right)$ is equal to $\sum_{\alpha \in A} D\left(z_{1}, \ldots, z_{n-2}, z_{\alpha}\right) \mathbf{1}_{z_{\alpha} U}\left(z_{n-1}\right)$, which we can always write as $\sum_{\alpha \in A} D\left(z_{1}, \ldots, z_{n-2}, z_{\alpha}\right) \delta_{U_{n}}^{1 / 2}\left(z_{n-1}\right) D_{\alpha}\left(z_{n-1}\right)$ with $D_{\alpha}=\delta_{U_{n}}^{-1 / 2} \mathbf{1}_{z_{\alpha} U}$ in $C_{c}^{\infty}\left(\operatorname{Lie}\left(Z_{n-1}\right)\right)$.

Each function $D\left(z_{1}, \ldots, z_{n-2}, z_{\alpha}\right)$ is equal to $W\left(z_{1}, \ldots, z_{\alpha}\right)-S\left(z_{1}, \ldots, z_{\alpha}\right)$, and the restrictions to $P_{n-1}$ of the functions $\delta_{U_{n}}^{-1 / 2}\left[\pi\left(z_{\alpha}\right) D\right]$ belong to the smooth submodule $\Phi^{-}(\pi)$ of $C^{\infty}\left(N_{n-1} \backslash P_{n-1}, \theta\right)$, which still satisfies the hypothesis of the statement.

Hence, by induction hypothesis, the function $D$ is a sum of functions of the form

$$
\prod_{k=1}^{n-1}\left[c_{i_{k}, k} \delta_{U_{k+1}}^{1 / 2} \ldots \delta_{U_{n}}^{1 / 2}\right]\left(z_{k}\right) v_{F}\left(z_{k}\right)^{m_{k}^{\prime}} \phi_{k}^{\prime}\left(z_{k}\right)
$$

for $i_{k}$ between 1 and $r_{k}$, null or positive integers or integer vectors $m_{k}^{\prime}$, and functions $\phi_{k}^{\prime}$ in $C_{c}^{\infty}\left(\operatorname{Lie}\left(Z_{k}\right)\right)$.

Now call $p$ the projection from $\pi_{(0)}$ to $\pi^{(1)}$. Then for any $a$ in $Z_{n-1}$, one has $\rho(a) p\left(\tilde{E}_{l}\right)=\sum_{k=1}^{q} M(a)_{k, l} p\left(\tilde{E}_{k}\right)$. Hence as $\rho(a) p\left(\tilde{E}_{l}\right)$ equals $p\left(\delta_{U_{n}}^{-1 / 2}(a) \pi_{(0)}(a) \tilde{E}_{l}\right)$, we deduce that there is a punctured neighbourhood of zero in $Z_{n-1}$ such that for each $l$, the function $\delta_{U_{n}}^{-1 / 2}(a) \pi_{(0)}(a) \tilde{E}_{l}-\sum_{k=1}^{q} M(a)_{k, l} \tilde{E}_{k}$ vanishes on elements $g=p a c$ of $G_{n-1}\left(p\right.$ in $P_{n-1}, a$ in $Z_{n-1}$, and $c$ in $\left.G_{n-1}\left(\mathfrak{O}_{F}\right)\right)$ such that $a$ is in this neighbourhood. 
In particular, there exists $N_{a}$ such that for every $\left(z_{1}, \ldots, z_{n-1}\right)$, the vector function

$$
\delta_{U_{n}}^{-1 / 2}(a) \pi_{(0)}(a) \tilde{E}\left(z_{1}, \ldots, z_{n-1}\right)-{ }^{t} M(a) \tilde{E}\left(z_{1}, \ldots, z_{n-1}\right)
$$

vanishes when we have $\left|z_{n-1}\right|_{F} \leq q_{F}^{-N_{a}}$.

This implies, as in the proof of Proposition 2.6 of $[\mathrm{C}-\mathrm{P}$, the following claim:

Claim. There is actually an $M^{\prime \prime}$ such that for every $z$ in $Z_{n-1}$, with $\left|z_{n-1}\right|_{F} \leq q_{F}^{-M^{\prime \prime}}$, and every $a$ in $Z_{n-1}$, with $|a|_{F} \leq 1$, the function $\tilde{E}\left(z_{1}, \ldots, z_{n-1} a\right)$ is equal to $\delta_{U_{n}}^{1 / 2}(a)^{t} M(a) \tilde{E}\left(z_{1}, \ldots, z_{n-1}\right)$.

Proof of the Claim. We denote $\left(z_{1}, \ldots, z_{n-2}\right)$ by $x$ and $z_{n-1}$ by $z$.

If $U$ is an open compact subgroup of $Z_{n-1}\left(\mathfrak{O}_{F}\right)$, such that $\tilde{E}$ and the homomorphism $a \in Z_{n-1} \mapsto M(a) \in G_{q}(\mathbb{C})$ are $U$ invariant, we denote by $u_{1}, \ldots, u_{s}$ the representatives of $Z_{n-1}\left(\mathfrak{O}_{F}\right) / U$ and by $\omega$ the canonical generator of $Z_{n-1} / Z_{n-1}\left(\mathfrak{O}_{F}\right)$. We put $M^{\prime \prime}=\max _{i, j}\left(N_{u_{i}}, N_{\omega}\right)$.

Then for $z$ in $\left\{z \in Z_{n-1},|z|_{F} \leq q_{F}^{-M^{\prime \prime}}\right\}$ and $a=\omega^{r} u_{i} u$ in $\left\{z \in Z_{n-1},|z|_{F} \leq 1\right\}$ (with $u$ in $U$, and $r \in \mathbb{N}$ ), we have

$$
\tilde{E}(x, z a)=\tilde{E}\left(x, z \omega^{r} u_{i}\right)=\delta_{U_{n}}^{1 / 2}\left(u_{i}\right)^{t} M\left(u_{i}\right) \tilde{E}\left(x, z \omega^{r}\right)
$$

because $z \omega^{r}$ belongs to $\left\{z \in Z_{n-1},|z|_{F} \leq q_{F}^{-M^{\prime \prime}}\right\} \subset\left\{z \in Z_{n-1},|z|_{F} \leq q_{F}^{-N_{u_{i}}}\right\}$. However, if $r \geq 1$, again one has

$$
\tilde{E}\left(x, z \omega^{r}\right)=\delta_{U_{n}}^{1 / 2}(\omega)^{t} M(\omega)\left(\omega_{i}\right) \tilde{E}\left(x, z \omega^{r-1}\right)
$$

and $z \omega^{r-1}$ belongs to

$$
\left\{z \in Z_{n-1},|z|_{F} \leq q_{F}^{-N_{2}}\right\} \subset\left\{z \in Z_{n-1},|z|_{F} \leq q_{F}^{-N_{\omega}}\right\},
$$

and repeating this step, we deduce the equality $\tilde{E}(x, z a)=\delta_{U_{n}}^{1 / 2}(a)^{t} M(a) \tilde{E}(x, z)$.

Hence there is an element $z_{0}$ in $Z_{n-1}$ with $\left|z_{0}\right|_{F}=q_{F}^{-M^{\prime \prime}}$ such that for every $\left(z_{1}, \ldots, z_{n-2}\right)$ in $Z_{1} \times \cdots \times Z_{n-2}$, the vector $\tilde{E}\left(z_{1}, \ldots, z_{n-1}\right)$ is equal to

$$
\delta_{U_{n}}^{1 / 2}\left(z_{n-1}\right)^{t} M\left(z_{n-1}\right)\left[\delta_{U_{n}}^{-1 / 2}\left(z_{0}\right)^{t} M\left(z_{0}^{-1}\right)\right] \tilde{E}\left(z_{1}, \ldots, z_{n-2}, z_{0}\right)
$$

for any $z_{n-1}$ with $\left|z_{n-1}\right|_{F} \leq 1$.

Hence the function $\mathbf{1}_{\left\{\left|z_{n-1}\right| \leq 1\right\}} S\left(z_{1}, \ldots, z_{n-1}\right)$ is equal to

$\left(x_{1}, \ldots, x_{q}\right)^{t} M\left(z_{n-1}\right)\left[\delta_{U_{n}}^{-1 / 2}\left(z_{0}\right)^{t} M\left(z_{0}^{-1}\right)\right] \times \tilde{E}\left(z_{1}, \ldots, z_{n-2}, z_{0}\right) \delta_{U_{n}}^{1 / 2}\left(z_{n-1}\right) \mathbf{1}_{\left\{\left|z_{n-1}\right| \leq 1\right\}}$.

One proves as for the function $D$ that function $\mathbf{1}_{\left\{\left|z_{n-1}\right|>1\right\}}\left(z_{n-1}\right) S\left(z_{1}, \ldots, z_{n-1}\right)$ is of the form

$$
\sum_{\beta \in B} S\left(z_{1}, \ldots, z_{n-2}, z_{\beta}\right) \delta_{U_{n}}^{1 / 2}\left(z_{n-1}\right) S_{\beta}\left(z_{n-1}\right)
$$

with $S_{\beta}$ in $C_{c}^{\infty}(F)$ for some finite set $B$.

By induction hypothesis again, applied to the function $\left(\delta_{U_{n}}^{-1 / 2} \tilde{E}_{i}\right)\left(z_{1}, \ldots, z_{n-2}, z_{0}\right)$ and the function $\left(\delta_{U_{n}}^{-1 / 2} S\right)\left(z_{1}, \ldots, z_{n-2}, z_{\beta}\right)$, we deduce that the function

$$
S=\mathbf{1}_{\left\{\left|z_{n-1}\right| \leq 1\right\}} S+\mathbf{1}_{\left\{\left|z_{n-1}\right|>1\right\}} S
$$

is a sum of functions of the form $\prod_{k=1}^{n-1} c_{i_{k}, n-k}\left(z_{k}\right) \delta_{U_{k+1}}^{1 / 2} \ldots \delta_{U_{n}}^{1 / 2}\left(z_{k}\right) v_{F}\left(z_{k}\right)^{m_{j}^{\prime \prime}} \phi_{k}^{\prime \prime}\left(z_{k}\right)$ for $i_{k}$ between 1 and $r_{k}$, null or positive integers or integer vectors $m_{k}^{\prime \prime}$, and functions $\phi_{k}^{\prime \prime}$ in $C_{c}^{\infty}(F)$. 
The statement follows as the function $W$ equals $D+S$.

\section{3. $L^{2}\left(Z_{n} N_{n} \backslash G_{n}\right)$ AND DISCRETE SERIES}

First we characterise the Whittaker functions which belong to $L^{2}\left(Z_{n} N_{n} \backslash G_{n}\right)$ in terms of exponents of the "shifted derivatives" (see [B, 7.2]). This result has been used in $\mathrm{M}$.

We say that a character of an $F^{*}$ is positive if its (complex) absolute value is of the form ||$_{F}^{r}$ for some positive real $r$.

Theorem 3.1. Let $\theta$ be a nondegenerate character of the group $N_{n}$, let $\pi$ be a $\theta$-generic representation of $G_{n}$, and let $c_{1, n-k}, \ldots, c_{r_{k}, n-k}$ be the characters of $Z_{k}$ appearing in a composition series of $\tau=\pi^{(n-k)}$. Then the integral

$$
\int_{N_{n} \backslash P_{n}}|W(p)|^{2} d p
$$

converges for any $W$ in $\pi$ if and only if all the characters $c_{i_{k}, k} \delta_{U_{k+1}}^{1 / 2}$ are positive for $k$ between 1 and $n-1$.

Proof. Again we prove the stronger statement:

Let $(\pi, V)$ be a $P_{n}$-submodule of $C^{\infty}\left(N_{n} \backslash P_{n}, \theta\right)$ such that for every $k$ between 1 and $n-1$, the $G_{k^{-m o d u l e}} \tau=\pi^{(n-k)}=\Psi^{-}\left(\Phi^{-}\right)^{n-k-1}(\pi)$ has a composition series such that, on each respective quotient, the central subgroup $Z_{k}$ acts by the characters $c_{1, n-k}, \ldots, c_{r_{k}, n-k}$. Then the integral

$$
\int_{N_{n} \backslash P_{n}}|W(p)|^{2} d p
$$

converges for any $W$ in $\pi$ if and only if all the characters $c_{i_{k}, k} \delta_{U_{k+1}}^{1 / 2}$ are positive for $k$ between 1 and $n-1$.

Let's first work out the proof for $G_{2}$, which already gives a good idea of the general case.

Suppose first that the characters $c_{i_{1}, 1} \delta_{U_{1}}^{1 / 2}$ are positive because of the equality $\int_{N_{2} \backslash P_{2}}|W(p)|^{2} d p=\int_{G_{1}}|W(g)|^{2} d g$, and, as $G_{1}$ is $Z_{1}$, the convergence of the integral is a straightforward consequence of Theorem 2.1.

Conversely suppose that the integral $\int_{N_{2} \backslash P_{2}}|W(p)|^{2} d p=\int_{Z_{1}}|W(z)|^{2} d z$ converges for every $W$ in $V$. Take a nonzero vector $v$ in $\Psi^{-}(V)$ that transforms by $c_{i_{1}, 1}$ under $Z_{1}$ (see Proposition 2.8). Then take a preimage $W$ of $v$ in $V$. According to Proposition 2.3, we deduce that for every $a$ in $Z_{1}$, the function $\delta_{U_{2}}^{-1 / 2}(a) \pi(a) W(z)-c_{i_{1}, 1}(a) W(z)$ vanishes when $z$ is in a punctured neighbourhood of zero depending on $a$. As in Claim 2 again, this implies that there exists an integer $N$ large enough such that $W(a z)$ equals $\delta_{U_{2}}^{1 / 2}(a) c_{i_{1}, 1}(a) W(z)$ for $|z|_{F} \leq q_{F}^{-N}$ and $|a|_{F} \leq 1$.

As $v$ is nonzero, the vector $W$ is not in the kernel of the projection from $V$ to $\Psi^{-}(V)$, so that according to Proposition 2.3, there is a $z_{0}$ of absolute value less than $q_{F}^{-N}$ such that $W\left(z_{0}\right)$ is not zero. Calling $W_{0}$ the function $\pi\left(z_{0}\right) W$, we deduce, as $W_{0}(z)$ equals $\delta_{U_{2}}^{1 / 2}(z) c_{i_{1}, 1}(z) W\left(z_{0}\right)$ for $z$ small enough, that the character $\delta_{U_{2}}^{1 / 2} c_{i_{1}, 1}$ must be positive in order to make the integral $\int_{Z_{1}}\left|W_{0}(z)\right|^{2} d z$ converge.

The general proof is by induction on $n$. As before, for $n=1$, the group $G_{1}$ is isomorphic to $F^{*}$, the groups $P_{1}$ and $N_{1}$ are trivial, and the statement is obvious. 
Now for $n \geq 2$, suppose first that all the characters $c_{i_{k}, k} \delta_{U_{k+1}}^{1 / 2}$ are positive. Let $W$ belong to the space of $\pi$; first we notice the equality

$$
\int_{N_{n} \backslash P_{n}}|W(p)|^{2} d p=\int_{N_{n-1} \backslash G_{n-1}}|W(g)|^{2} d g .
$$

Now the Iwasawa decomposition reduces the convergence of this integral to that of

$$
\int_{A_{n-1}}|W(a)|^{2} \delta_{N_{n-1}}^{-1}(a) d^{*} a
$$

Using coordinates $\left(z_{1}, \ldots, z_{n-1}\right)$ (see Lemma 1.1) of $A_{n-1}$, the function $\delta_{N_{n-1}}^{-1}\left(z_{1}, \ldots, z_{n-1}\right)$ is equal to $\prod_{k=1}^{n-2}\left(\delta_{U_{k+1}} \ldots \delta_{U_{n-1}}\right)^{-1}\left(z_{k}\right)$.

According to Theorem 2.1 the function $\left|W\left(z_{1}, \ldots, z_{n-1}\right)\right|^{2}$ is bounded by a sum of functions of the form

$$
\prod_{k=1}^{n-1}\left|c_{i_{k}, k}\right|\left(z_{k}\right)\left|c_{l_{k}, k}\right|\left(z_{j}\right)\left(\delta_{U_{k}+1} \ldots \delta_{U_{n}}\right)\left(z_{k}\right) v_{F}\left(z_{k}\right)^{m_{k}} \phi_{k}\left(z_{k}\right) .
$$

Hence our integral will converge if the same is true of the integrals

$$
\int_{A_{n-1}} \prod_{k=1}^{n-1}\left|c_{i_{k}, k}\right|\left(z_{k}\right)\left|c_{l_{k}, k}\right| \delta_{U_{n}}\left(z_{k}\right) v_{F}\left(t_{k}\right)^{m_{k}} \phi_{k}\left(z_{k}\right) d z_{1} \ldots d z_{n}
$$

i.e. if the integrals $\int_{Z_{k}}\left|c_{i_{k}, k}\right|\left(z_{k}\right)\left|c_{l_{k}, k}\right|\left(z_{k}\right) \delta_{U_{n}}\left(z_{k}\right) v_{F}\left(t_{k}\right)^{m_{k}} \phi_{k}\left(t_{k}\right) d z_{k}$ converge for any $k$ between 1 and $n-1$.

But the restriction of $\delta_{U_{n}}$ to $Z_{k}$ is equal to $\delta_{U_{k+1}}$, so the convergence follows from our assertion on the characters $c_{i_{k}, k} \delta_{U_{n}}^{1 / 2}$.

Conversely, suppose that every $W$ in $\pi_{(0)}$ belongs to the space $L^{2}\left(N_{n} \backslash P_{n}\right)$ corresponding to a right invariant measure on $N_{n} \backslash P_{n}$.

By Iwasawa decomposition, one obtains that

$$
\int_{N_{n} \backslash P_{n}}|W(p)|^{2} d p=\int_{N_{n-1} \backslash G_{n-1}}|W(g)|^{2} d g
$$

is equal to $\int_{A_{n-1} \times F_{n}}|W(a k)|^{2} \delta_{N_{n-1}}^{-1}(a) d^{*} a d k$ which is greater than $d k(U) \int_{A_{n-1}}|W(a)|^{2} \delta_{N_{n-1}}^{-1}(a) d^{*} a$ for some compact open subgroup $U$ fixing $W$. In particular the integral $\int_{A_{n-1}}|W(a)|^{2} \delta_{N_{n-1}}^{-1}(a) d^{*} a$ converges for any $W$ in $\pi$.

This, by Fubini's theorem and the smoothness of $W$, implies that $\int_{A_{n-2}}|W(a)|^{2} \delta_{N_{n-1}}^{-1}(a) d^{*} a$ is finite for any $W$ in $\pi$. However, the restriction of $\delta_{N_{n-1}}$ to $A_{n-1}$ is equal to $\delta_{N_{n-2}} \delta_{U_{n-1}}$, so that the integral $\int_{A_{n-2}}|W(a)|^{2} \delta_{U_{n-1}}^{-1} \delta_{N_{n-2}}^{-1}(a) d^{*} a$ is finite for $W$ in $\pi$, which by Iwasawa decomposition again implies that $\int_{N_{n-1} \backslash P_{n-1}}\left|\delta_{U_{n-1}}^{-1 / 2} W(p)\right|^{2} d p$ is finite.

The functions $\delta_{U_{n-1}}^{-1 / 2} W$ belong to the space of $\phi^{-}(\pi)$. Hence by induction, all the characters $c_{i_{k}, k} \delta_{U_{k+1}}^{1 / 2}$ are positive for $k \leq n-2$, so we only need to check that the characters $c_{i_{n-1}, n-1} \delta_{U_{n}}^{1 / 2}$ are positive. Suppose that one of them, $c_{1, n-1} \delta_{U_{n}}^{1 / 2}$ for instance, wasn't positive.

Then, taking $v$ nonzero in $\Psi^{-}(\pi)$ such that $Z_{n-1}$ multiplies $v$ by $c_{1, n-1}$, according to Proposition 2.3 and taking $W$ as a preimage of $v$ in $\pi$, there is a positive integer $N_{a}$ such that $\delta_{U_{n}}^{-1 / 2}(a) \pi(a) W(g)-c_{1, n-1}(a) W(g)$ is zero whenever there is any $g$ 
in $G_{n-1}$ with $|z(g)|_{F} \leq q_{F}^{-N_{a}}$. As in Claim 2, this implies that there is a positive integer $N$ such that $W(a g)$ is equal to $\delta_{U_{n}}^{1 / 2}(a) c_{1, n-1}(a) W(g)$ whenever $|z(g)|_{F} \leq$ $q_{F}^{-N}$ and $|a|_{F} \leq 1$. We recall that $W$ doesn't belong to $V\left(U_{n}, 1\right)$ (otherwise $v$ would be zero), hence according to Proposition 2.3, there is $g_{0}$ in $G_{n-1}$ with $\left|z\left(g_{0}\right)\right|_{F} \leq$ $q_{F}^{-N}$ such that $W\left(g_{0}\right)$ is nonzero. We denote by $W_{0}$ the function $\pi\left(g_{0}\right) W$, and we recall that the integral

$$
\begin{aligned}
& \int_{A_{n-1}}\left|W_{0}(a)\right|^{2} \delta_{N_{n-1}}^{-1}(a) d^{*} a \\
& \quad=\int_{Z_{1} \times \cdots \times Z_{n-1}}\left|W_{0}\left(z_{1} \ldots z_{n-1}\right)\right|^{2} \delta_{N_{n-1}}^{-1}\left(z_{1} \ldots z_{n-1}\right) d z_{1} \ldots d z_{n-1}
\end{aligned}
$$

is finite. Hence the smoothness of $W_{0}$ and Fubini's theorem imply that the integral

$$
\int_{Z_{n-1}}\left|W_{0}\left(z_{n-1}\right)\right|^{2} \delta_{N_{n-1}}^{-1}\left(z_{n-1}\right) d z_{n-1}=\int_{Z_{n-1}}\left|W_{0}\left(z_{n-1}\right)\right|^{2} d z_{n-1}
$$

is finite. However, for $\left|z_{n-1}\right|_{F} \leq 1$, the function $W_{0}\left(z_{n-1}\right)$ is equal to $\delta_{U_{n}}^{1 / 2}\left(z_{n-1}\right) c_{1, n-1}\left(z_{n-1}\right) W\left(g_{0}\right)$ with $W\left(g_{0}\right)$ nonzero. Hence it is square integrable at zero if and only if $\delta_{U_{n}} c_{1, n-1}^{2}$, thus $\delta_{U_{n}}^{1 / 2} c_{1, n-1}$ is positive.

Remark 3.1. The last proof more or less contains the following fact (which is more precisely a consequence of an induction, and the last step of the proof):

For every character $c_{i_{k}, n-k}$ appearing in a factor series of $\pi^{(n-k)}$, there is $W$ in $V$ such that $W\left(z_{k}\right)$ is equal to $\left[c_{i_{k}, n-k} \delta_{U_{k+1}}^{1 / 2} \ldots \delta_{U_{n}}^{1 / 2}\right]\left(z_{k}\right)$ near zero. Hence this family of characters is minimal in the sense that each of them must occur in the expansion given in Proposition 2.1 of some $W$ in $V$.

From this we deduce a characterization of the Whittaker functions in $L^{2}\left(Z_{n} N_{n} \backslash G_{n}\right)$.

Corollary 3.1. Let $\theta$ be a nondegenerate character of the group $N_{n}$, let $\pi$ be a $\theta$-generic representation of $G_{n}$ with unitary central character, and let $c_{1, n-k}, \ldots$, $c_{r_{k}, n-k}$ be the central characters appearing in the factor series of $\tau=\pi^{(n-k)}$. Then the integral

$$
\int_{Z_{n} N_{n} \backslash G_{n}}|W(g)|^{2} d g
$$

converges for any $W$ in $\pi$ if and only if all the characters $c_{i_{k}, k}$ are positive for $k$ between 1 and $n-1$.

Proof. By the Iwasawa decomposition, the integral $\int_{Z_{n} N_{n} \backslash G_{n}}|W(g)|^{2} d g$ converges for every $W$ in $W(\pi, \theta)$ if and only if $\int_{A_{n-1}}|W(a)|^{2} \delta_{N_{n}}^{-1}(a) d a$ converges for every $W$ in $W(\pi, \theta)$.

As the character $\delta_{N_{n}}$ restricts to $G_{n-1}$ as $\delta_{N_{n-1}} \delta_{U_{n}}$, this integral is equal to

$$
\int_{A_{n-1}}\left|\delta_{U_{n}}^{-1 / 2} W(a)\right|^{2} \delta_{N_{n-1}}^{-1}(a) d a .
$$


However, this integral converges for any $W$ in $W(\pi, \theta)$ if and only if so does the integral

$$
\int_{N_{n} \backslash P_{n}}\left|\delta_{U_{n}}^{-1 / 2} W(p)\right|^{2} d p
$$

for any $W$ in $W(\pi, \theta)$.

By the statement in the proof of Theorem 3.1 applied to $\delta_{U_{n}}^{-1 / 2} \otimes \pi^{\prime}$, this is the case if and only all the characters $c_{i_{k}, k}$ are positive for $k$ between 1 and $n-1$.

Let $P$ be a standard proper parabolic subgroup of $G_{n}, U$ its unipotent radical, and $M$ its standard Levi subgroup. If $(\pi, V)$ is a smooth irreducible representation of $G_{n}$, one refers to a cuspidal exponent of $\pi$ with respect to $P$ as a character $\chi$ of the center of $M$ such that the characteristic space of the Jacquet module $\left(V_{U}\right)_{\chi, \infty}$ is nonzero. Denoting by $\Delta$ the set of simple roots $\left\{\alpha_{1}, \ldots, \alpha_{n}\right\}$ of $G_{n}$, we denote by $P^{\left\{i_{1}, \ldots, i_{t}\right\}}$ the standard parabolic subgroup associated with the set of positive roots $\Delta-\left\{\alpha_{i_{1}}, \ldots, \alpha_{i_{t}}\right\}$, by $U^{\left\{i_{1}, \ldots, i_{t}\right\}}$ its unipotent radical, and by $M^{\left\{i_{1}, \ldots, i_{t}\right\}}$ its standard Levi subgroup, which admits as a central subgroup the product $Z_{i_{1}} \ldots Z_{i_{t}}$.

Notice that except for Case $\mathrm{D}$, for $\left\{i_{1}, \ldots, i_{t}\right\}=\{2\}$, where we used the notation $U_{2, n-2}$ for $U^{\{1,2\}}$, the group $U^{\{k\}}$ is what we already denoted by $U_{k, n-k}$.

We denote by $A_{i_{1}, \ldots, i_{t}}^{-}$the set

$$
\left\{z_{i_{1}} \ldots z_{i_{t}} \in Z_{i_{1}} \ldots Z_{i_{t}},\left|z_{i_{k}}\right|_{F} \leq 1 \text {, and }\left|z_{i_{1}} \ldots z_{i_{t}}\right|_{F}<1\right\} \text {. }
$$

Theorem 4.4.6 of [C] then asserts that $\pi$ with unitary central character is a discrete series representation if and only if, for every standard parabolic subgroup $P^{\left\{i_{1}, \ldots, i_{t}\right\}}$, if $\chi$ is a cuspidal exponent of $\pi$ with respect to $P^{\left\{i_{1}, \ldots, i_{t}\right\}}$, the restriction of $\chi$ to $A_{i_{1}, \ldots, i_{t}}^{-}$is less than 1 , or equivalently if $\chi$ restricted to $Z_{i_{1}} \ldots Z_{i_{t}}$ is positive.

We also notice that for any $k$, the Jacquet module $V_{U\left\{i_{k}\right\}}$ surjects onto $V_{U\left\{i_{1}, \ldots, i_{t}\right\}}$ and the character $\delta_{U\left\{i_{1}, \ldots, i_{t}\right\}}$ restricts to $Z_{i_{k}}$ as $\delta_{U\left\{i_{k}\right\}}$. Hence if $\chi$ is a cuspidal exponent of $\pi$ with respect to $P^{\left\{i_{1}, \ldots, i_{t}\right\}}$, then $\chi_{\mid Z_{i_{k}}}$ is the restriction to $Z_{i_{k}}$ of a cuspidal exponent of $\pi$ with respect to $P^{\left\{i_{k}\right\}}$. This implies that $\pi$ irreducible with unitary central character is a discrete series representation if and only if the cuspidal exponents of $\pi$ with respect to maximal parabolic subgroups $P^{\left\{i_{k}\right\}}$ have positive restriction to $Z_{i_{k}}$.

We call a character $\chi$ of $Z_{k}$, such that $\left(V^{(n-k)}\right)_{\chi}$ (or equivalently $\left(V^{(n-k)}\right)_{\chi, \infty}$ ) is nonzero, an exponent of the derivative $\left(\pi^{(n-k)}, V^{(n-k)}\right)$. Now we recall that we showed in Proposition 2.7 that the $Z_{k}$ modules $V_{U_{k, n-k}}$ and $V^{(n-k)}$ have the same nonzero weight subspaces. This allows us to prove in our four cases the following conjecture of Lapid and Mao ([L-M, Conjecture 3.5]).

Patrick Delorme proved this conjecture for general split groups (D2, Theorem 8]).

Theorem 3.2. Let $\pi$ be a generic representation of $G_{n}$ with unitary central character and with Whittaker model $W(\pi, \theta)$. Then the following statements are equivalent:

i) The integral

$$
\int_{N_{n} Z_{n} \backslash G_{n}}|W(g)|^{2} d g
$$

converges for any $W$ in $W(\pi, \theta)$. 
ii) All the exponents of the derivatives of $\pi$ are positive.

iii) the representation $\pi$ is square-integrable.

Proof. By assumption, the exponents of the derivatives of $\pi$ are the characters $c_{i_{k}, k}$ of Corollary 3.1. Hence i) $\Leftrightarrow$ ii) is Corollary 3.1.

ii) $\Leftrightarrow$ iii): We treat Case D separately, so assume first that $G_{n}$ is not $G S O(2(n-$ $1), F)$.

By Proposition 2.6. every cuspidal exponent of $\pi$ corresponding to $V_{U^{\{k\}}}$ is positive if and only if every exponent of the derivative $\pi^{(n-k)}$ is positive. However, we have already seen that this implies that $\pi$ is a discrete series representation.

For Case D, we could have reversed the roles of the roots $\alpha_{1}$ and $\alpha_{2}$ (which correspond to the two symmetric roots at the end of the Dynkin diagram). The only effect it would have is to change the definition of the derivative functors $\pi^{(n-2)}$ and $\pi^{(n-1)}$. Indeed $U_{2}$ would become $U_{\alpha_{2}}$, and $Z_{1}$ and $Z_{2}$ would be exchanged. The character $\theta_{3}$ would have to be trivial on $U_{\alpha_{2}}$ instead of being trivial on $U_{\alpha_{1}}$. But i) and ii) would still be equivalent in this case, and i) is independent of these choices.

In both cases, the maps $I_{k}$ from $V_{U_{k, n-k}}$ to $V^{(n-k)}$ take nonzero weight subspaces to nonzero weight subspaces. For $n \geq 3$, the space $V_{U_{k, n-k}}$ is equal to $V_{U^{\{k\}}}$. In the first case, $V_{U_{1, n-1}}$ is equal to $V_{U^{\{1\}}}=V_{U_{\alpha_{1}}}$, and it is equal to $V_{U^{\{2\}}}=V_{U_{\alpha_{2}}}$ in the second case. This implies that all the exponents of the derivatives of $\pi$ are positive if and only if all cuspidal exponents of $\pi$ with respect to maximal parabolic subgroups are positive. Again this proves ii) $\Leftrightarrow$ iii).

\section{REFERENCES}

[B] J. N. Bernstein, P-invariant distributions on $G L(N)$ and the classification of unitary representations of $G L(N)$ (non-Archimedean case), Lecture Notes in Math., vol. 1041, Springer-Verlag, Berlin, 1983, 50-102. MR748505 (86b:22028)

[B-Z] J. N. Bernstein and A.V. Zelevinsky, Representations of the group $G L(n, F)$ where $F$ is a local non-archimedean field, Russian Math. Surveys, 31:3 (1976), 1-68. MR0425030 $(54: 12988)$

[B-Z.2] J. N. Bernstein and A.V. Zelevinsky, Induced representations of reductive p-adic groups, Ann. Sc. E.N.S., 1977.

[Bu] C. Bushnell, Representations of reductive p-adic groups: Localization of Hecke algebras and applications, J. London Math. Soc., 63 (2001), 364-386. MR1810135 (2001m:22034)

[B-H] C. Bushnell and G. Henniart, On the derived subgroups of certain unipotent subgroups of reductive groups over infinite fields, Transformation groups 7 (2002), no. 3, pp. 211-230. MR.1923971 (2003h:20084)

[C] W. Casselman, Introduction to the theory of admissible representations of p-adic reductive groups, http://www.math.ubc.ca/ cass/research.html.

[C-S] W. Casselman and J. Shalika, The unramified principal series of p-adic groups II. The Whittaker function, Compositio Math. 41 (1980), 207-231. MR581582 (83i:22027)

[C-P] J. W. Cogdell and I. I. Piatetski-Shapiro, Derivatives and L-functions for $G L(n)$, to appear in The Heritage of B. Moishezon, IMCP.

[D] P. Delorme, Constant term of $H_{\psi}$-spherical functions on a reductive $p$-adic group, Transactions American Math. Soc. 362 (2010), 933-955. MR2551511(2011d:22016)

[D2] P. Delorme, Théorème de Paley-Wiener pour les fonctions de Whittaker sur un groupe réductif p-adique, Preprint.

[J] H. Jacquet, Generic representations, in Non-commutative harmonic analysis, MarseilleLuminy, 1976, Springer-Verlag, Lecture Notes No. 587 (1976), 376-378. MR0499005 $(58: 16985)$

[L-M] E. Lapid and Z. Mao On the asymptotics of Whittaker functions, Represent. Theory 13 (2009), 63-81. MR2495561 (2010b:22024) 
[M] N. Matringe, Distinction of some induced representations, Math. Res. Lett., 2010, vol. 17, no. 1, 77-97. MR2592729

[R] F. Rodier, Modèle de Whittaker des représentations admissibles des groupes réductifs $p$ adiques déployés, C. R. Acad. Sci. Paris Ser. A-B 275, A1045-A1048, 1972. MR0315053 $(47: 3602)$

[Z] A.V. Zelevinsky, Induced representations of reductive p-adic groups II, Ann. Sc. E.N.S., 1980.

School of Mathematics, University of East Anglia, Norwich, NR4 7TJ, United KingDOM

Current address: Laboratoire de Mathématiques et Applications, Université de Poitiers, Téléport 2 - BP 30179, Boulevard Marie et Pierre Curie, 86962, Futuroscope Chasseneuil Cedex, France

E-mail address: Nadir.Matringe@math.univ-poitiers.fr 